\title{
Genome-wide characterization and expression analyses of the MYB superfamily genes during developmental stages in Chinese jujube
}

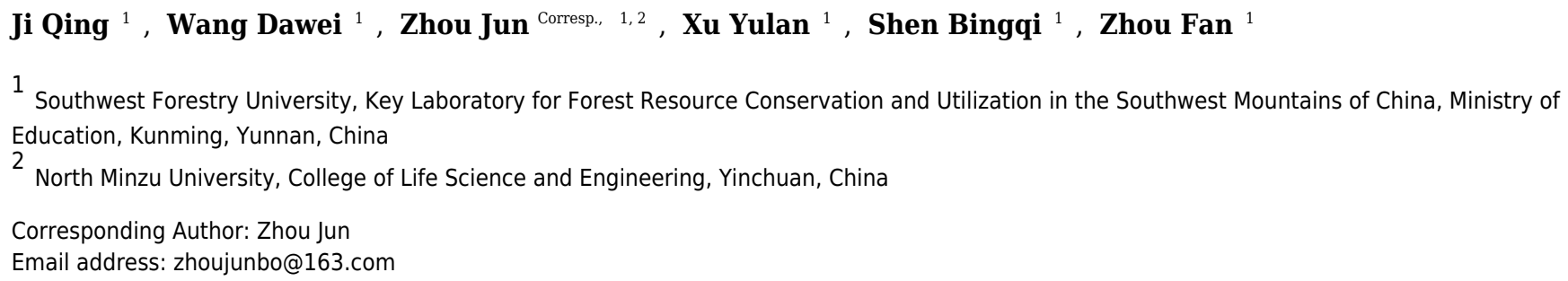

The MYB transcription factor superfamily, one of the largest gene superfamilies, regulates a variety of physiological processes in plants. Although many MYB superfamily genes have been identified in plants, the MYB TFs in Chinese jujube (Ziziphus jujuba Mill.) have not been fully identified and characterized. Additionally, the functions of these genes remain unclear. In total, we identified 171 MYB superfamily genes in jujube and divided them into 5 subfamilies containing 99 genes of the R2R3-MYB subfamily, 58 genes of the MYBrelated subfamily, 4 genes of the R1R2R3-MYB subfamily, 1 gene of the 4R-MYB subfamily, and 9 genes of the atypical MYB subfamily. The 99 R2R3-MYB genes of jujube were divided into 35 groups, $\mathrm{C} 1$ to $\mathrm{C} 35$, and the 58 MYB-related genes were divided into the following groups: the R-R-type, CCA1-like, I-box-binding-like, TBP-like, CPC-like, and Chinese jujubespecific groups. ZjMYB genes in jujube were well supported by additional highly conserved motifs and exon/intron structures. Most R1 repeats of MYB-related proteins comprised the $R 2$ repeat and had highly conserved EED and EEE residue groups in jujube. Three tandem duplicated gene pairs were found on twelve chromosomes in jujube. According to an expression analysis of 126 ZjMYB genes, MYB-related genes played important roles in jujube development and fruit-related biological processes. The total flavonoid content of jujube fruit decreased as ripening progressed. Ninety-three expressed genes were identified in the RNA-sequencing data from jujube fruit, and 56 ZjMYB members presented significant correlations with total flavonoid contents by correlation analysis. Five pairs of paralogous MYB genes within jujube were composed of 9 jujube MYB genes. Fourteen ZjMYB genes had the same homology to the MYB genes of Arabidopsis and peach, indicating that these 14 MYB genes and their orthologs probably existed before the ancestral divergence of the MYB superfamily. We used a synteny analysis of MYB genes between jujube and Arabidopsis to predict that the functions of the ZjMYBs involve 
flavonoid/phenylpropanoid metabolism, the light signaling pathway, auxin signal transduction, and responses to various abiotic stresses (cold, drought and salt stresses). Additionally, we speculate that ZjMYB108 is an important transcription factor involved in the flavonoid metabolic pathway. This comprehensive analysis of MYB superfamily genes in jujube lay a solid foundation for future comprehensive analyses of ZjMYB gene functions. 
1 Genome-wide characterization and expression analyses of the

2 MYB superfamily genes during developmental stages in

3 Chinese jujube

4 Ji Qing ${ }^{1}$, Wang Dawei ${ }^{1}$, Zhou Jun ${ }^{1,2}$, Xu Yulan ${ }^{1}$, Shen Bingqi ${ }^{1}$, Zhou Fan ${ }^{1}$

$5 \quad{ }^{1}$ Key Laboratory for Forest Resource Conservation and Utilization in the Southwest Mountains of China,

6 Ministry of Education, Southwest Forestry University, Kunming, Yunnan, China

$7 \quad{ }^{2}$ College of Life Science and Engineering, North Minzu University, Yinchuan, China

8

9 Corresponding Author:

10 Zhou Jun ${ }^{1,2}$

11 NO. 300, Bailong Temple, Panlong District, Kunming, 650224, Yunnan, China 


\section{Abstract}

The MYB transcription factor superfamily, one of the largest gene superfamilies, regulates a variety of physiological processes in plants. Although many MYB superfamily genes have been identified in plants, the MYB TFs in Chinese jujube (Ziziphus jujuba Mill.) have not been fully identified and characterized. Additionally, the functions of these genes remain unclear. In total, we identified $171 M Y B$ superfamily genes in jujube and divided them into 5 subfamilies containing 99 genes of the R2R3-MYB subfamily, 58 genes of the MYB-related subfamily, 4 genes of the R1R2R3-MYB subfamily, 1 gene of the 4R-MYB subfamily, and 9 genes of the atypical MYB subfamily. The 99 R2R3-MYB genes of jujube were divided into 35 groups, C1 to C35, and the 58 MYB-related genes were divided into the following groups: the R-R-type, CCA1-like, I-boxbinding-like, TBP-like, CPC-like, and Chinese jujube-specific groups. ZjMYB genes in jujube were well supported by additional highly conserved motifs and exon/intron structures. Most R1 repeats of MYB-related proteins comprised the R2 repeat and had highly conserved EED and EEE residue groups in jujube. Three tandem duplicated gene pairs were found on twelve chromosomes in jujube. According to an expression analysis of 126 ZjMYB genes, MYB-related genes played important roles in jujube development and fruit-related biological processes. The total flavonoid content of jujube fruit decreased as ripening progressed. Ninety-three expressed genes were identified in the RNA-sequencing data from jujube fruit, and 56 ZjMYB members presented significant correlations with total flavonoid contents by correlation analysis. Five pairs of paralogous $M Y B$ genes within jujube were composed of 9 jujube $M Y B$ genes. Fourteen $Z j M Y B$ genes had the same homology to the $M Y B$ genes of Arabidopsis and peach, indicating that these $14 M Y B$ genes and their orthologs probably existed before the ancestral divergence of the MYB superfamily. We used a synteny analysis of $M Y B$ genes between jujube and Arabidopsis to predict that the functions of the ZjMYBs involve flavonoid/phenylpropanoid metabolism, the light signaling pathway, auxin signal transduction, and responses to various abiotic stresses (cold, drought and salt stresses). Additionally, we speculate that ZjMYB108 is an important transcription factor involved in the flavonoid metabolic pathway. This comprehensive analysis of $M Y B$ 
superfamily genes in jujube lay a solid foundation for future comprehensive analyses of $Z j M Y B$ gene functions.

5

\section{Introduction}

Transcription factors (TFs) play essential roles in plants by controlling the expression of genes, activating or inhibiting the transcription of other genes, or interacting with other TFs to regulate gene transcription (Singh, Foley \& Onate-Sanchez, 2002; Liu, White \& MacRae, 1999). The MYB family is a large TF family present in all eukaryotes, and MYBs regulate a variety of physiological processes in plants (Riechmann et al., 2000). The first identified MYB gene was $v-m y b$ in 1982 from the avian myeloblastosis virus, a chicken oncogene that leads to acute myeloblastic leukemia (Klempnauer et al., 1982), thus leading to the name myb. C-myb, a v-MYB-related gene was subsequently found in animal cells. Corresponding genes were also identified in human tumor cells (A-MYB and B-MYB) at the same time and these genes were found to modulate cell proliferation, differentiation, and apoptosis (Weston, 1998). The first identified MYB gene of a plant was C1, which was isolated from Zea mays (Paz-Ares et al., 1987) and is involved in anthocyanin biosynthesis. As the Arabidopsis MYB gene family has gradually been identified (Stracke, Werber \& Weisshaar, 2001; Chen et al., 2006), more MYB genes have been identified in many other plants (Zhang \& Ma et al., 2018; Li X et al., 2016; Hou et al., 2014; Zhou et al., 2015); these genes are identified by the family-specific feature of a highly conserved MYB domain at the N-terminus (Lipsick, 1996; Mmadi et al., 2017; Dubos et al., 2010). This domain gene usually comprises 1-4 imperfect amino acid sequence repeats (R1-R4) of approximately 50-53 amino acids (Dubos et al., 2010; Mmadi et al., 2017), with each forming three $\alpha$-helices, and the second and third helices form a helix-turn-helix (HTH) motif (Ogata et al., 1996; Dubos et al., 2010). MYB genes are classified into the following subfamilies according to the number of MYB imperfect tandem repeats (Rs) of the proteins: MYB-related (or 1R-MYB, one R), R2R3-MYB (two Rs), R1R2R3MYB (three Rs), and 4R-MYB (four Rs) (Dubos et al., 2010; Zhang \& Ma et al., 2018).

Many studies have shown that MYB TFs are involved in physiological and biochemical processes 
80 in plants and responses to various biotic as well as abiotic stresses (Abe et al., 2003; Agarwal et al., 2006; Andrew et al., 2007; Cominelli \& Tonelli, 2009; Raffaele et al., 2008), and the function of MYB has also been studied in detail in some MYB proteins. For example, MYB7 plays a role in kiwifruit (Actinidia chinensis) through transcriptional activation of metabolic pathway genes to modulate carotenoid and Chl pigment accumulation in tissues (Ampomah--Dwamena et al., 2018). The AtDIV2 of the R-R-type MYB gene in Arabidopsis is required for ABA signaling and plays a negative role in salt stress (Fang et al., 2018). In tobacco, overexpression of the PbrMYB5 gene enhanced tolerance to chilling stresses (Xing et al., 2018). In tomato (Solanum lycopersicum L.) plants, the R3-MYB gene is involved in a feedback mechanism, and if it is activated by endogenous or exogenous stimuli, anthocyanin production is inhibited (Colanero, Perata \& Gonzali, 2018). During continuous light treatments, the levels of McMYB10 increased and promoted the expression levels of McCOP1-1 and McCOP1-2 as well as anthocyanin biosynthesis in crabapple ( $\mathrm{Li}$ et al., 2018). MYB TFs are also involved in flavonoid/phenylpropanoid metabolism; for example, overexpression of the AtMYB12 gene of Arabidopsis enhanced the accumulation of flavonoid content under low temperatures in a light-dependent manner (Bhatia et al., 2018). During strawberry ripening, the $M Y B 10$ gene in Fragaria $\times$ ananassa plays a general regulatory role in the flavonoid/phenylpropanoid pathway (Puche et al., 2014). In addition, ATMYB12 (Bhatia et al., 2018), ATMYB018 (Ballesteros et al., 2001), ATMYB21 (Shin et al., 2002), ATMYB075 and ATMYB090 (Li et al., 2006) genes are involved in the light signaling pathway in Arabidopsis. R2R3-MYB TF family members in petunia (Petunia hybrida) are developmentally and environmentally regulated to control complex floral and vegetative pigmentation patterning (Albert et al., 2011).

Chinese jujube (Ziziphus jujuba Mill.) is a traditional economic tree species in China. Jujube has flourished for a long time because of its strong resistance, simple management, high yield, rich nutrition, and good economic and ecological benefits (Li et al., 2007; Zhao, Liu \& Tu, 2008). Many consumers favor jujube because of its good taste, rich nutrition, powerful health functions and alternative medicinal properties (Wang \& Xu et al., 2018; Zhang \& Bian et al., 2018; Lam et 
107

108

109

110

111

112

113

114

115

116

117

118

119

120

121

122

123

124

125

126

127

128

129

130

131

al., 2016). Fresh jujube fruits develop rapidly particularly in the high-quality cultivar 'Dongzao' (Yuan et al., 2017). The entire Chinese jujube genome sequence was obtained in 2014 (Liu et al., 2014), providing data for genome-wide analyses of the MYB superfamily. Although many studies have shown that MYB TFs are involved in physiological and biochemical processes in plants, the MYB TFs of jujube have not been fully identified and characterized, and the expression of $M Y B$ genes in the different developmental stages of fresh jujube fruit remains unclear. Thus, in this study, the MYB superfamily members in jujube were analyzed and identified, and their protein physicochemical properties, motif and exon/intron composition, correlation with flavonoid content, syntenic relationships, and expression levels in different developmental stages of fresh jujube were analyzed. These findings should inform the characterization of ZjMYB genes, and this study will be helpful for future functional studies of the $Z j M Y B$ superfamily genes involved in fruit development in jujube.

\section{Materials and methods}

\section{Plant materials}

Twelve-year-old Chinese jujube served as test materials at the 'Xuefeng' ecological park of Gengjiaying township, Yiliang county in Kunming city of Yunnan province, China, which is the teaching experiment base of Southwest Forestry University. Fruits were selected from five periods, including the young stage, enlargement stage, white mature stage, half-red stage, and full-red stage. The samples of these five periods were harvested in turn 25, 39, 74, 83 and 99 days after anthesis. These fruits were immediately frozen in liquid nitrogen and stored at $-80^{\circ} \mathrm{C}$. Three biological repeats were performed for each developmental stage for RNA-sequencing, total flavonoid content determination, and qRT-PCR analysis.

Jujube $M Y B$ superfamily gene identification 
132 The completed jujube genome sequence and chromosome information were identified

133 from the DDBJ/EMBL/Gen-Bank (accession JREP00000000), which provides Chinese jujube

134 genome data (Liu et al., 2014). The Pfam database (http://pfam.xfam.org/) was searched to obtain

135 the hidden Markov model (HMM) profile for the MYB binding domain (PF00249) (Finn et al.,

136 2016; Zhang \& Ma et al., 2018), and all putative MYB genes were obtained from the jujube

137 genome database (Liu et al., 2014). The presence of a MYB domain in the selected MYB proteins

138 was further verified using the online program SMART (http://smart.embl-heidelberg.de/) and

139 HMMER (https://www.ebi.ac.uk/Tools/hmmer/). Next, manual analysis was performed using the

140 ClustalX program to confirm MYB conserved domains or motifs. Ultimately, we confirmed that

141 the genes containing the MYB domain were members of the MYB superfamily. Based on previous

142 research, we downloaded 198 MYB family protein sequences and chromosome information in 143 Arabidopsis from The Arabidopsis Information Resource (TAIR) (http://www.Arabidopsis.org/)

144 (Stracke, Werber \& Weisshaar, 2001; Chen et al., 2006).

145

146

147

148

149

150

151

152

153

154

155

156

157

Multiple sequence alignment and phylogenetic tree construction of MYB

\section{proteins}

In this study, we will refer to the classification of the Arabidopsis MYB gene family and the results of a phylogenetic tree for subfamily classification of the jujube MYB family and grouping (Dubos et al., 2010; Chen et al., 2006; Zhang \& Ma et al., 2018). We constructed the first phylogenetic tree with 126 R2R3-MYB proteins, 5 R1R2R3-MYB proteins and 14 R-MYB protein of Arabidopsis and 171 MYB family proteins of jujube. We used the ClustalW program for multiple comparisons of amino acid sequences. The neighbor-joining (NJ) phylogenetic tree was constructed by 1,000 repeated bootstrap analyses using MEGA 6 software (Tamura et al., 2013). Genes not belonging to R2R3-MYB, R1R2R3-MYB and 4R-MYB in the first phylogenetic tree of MYB genes were used to construct a second phylogenetic tree with the $601 \mathrm{R}-\mathrm{MYB}$ proteins and 6 atypical MYB proteins in Arabidopsis. Phylogenetic tree construction parameters reference 
the first phylogenetic tree.

\section{Gene structure and conserved motif analysis}

The exon-intron structures of ZjMYB genes were generated with the Gene Structure Display Server (GSDS: http://gsds.cbi.pku.edu.cn/) (Hu et al., 2015). The conserved motifs of ZjMYB proteins were defined using the MEME program (http://meme-suite.org/tools/meme) (Bailey et al., 2009). The following parameter settings were used: distribution of motifs, 0 or 1 per sequence; the minimum width and maximum width of motifs, 6 and 250, respectively; maximum number of motifs to find, 15; and default parameters. The conserved domains of $Z j M Y B$ proteins were defined using Pfam (http://pfam.xfam.org/). We visualized the Pfam, MEME and GSDS results using TBTOOLS software (http://cj-chen.github.io/TBtools/) (Chen et al., 2018; Liu et al., 2017). The ExPASy online tool (http://www.expasy.ch/tools/protparam.html) was used to analyze the physiological and biochemical characteristics of the $Z j M Y B$ genes.

\section{Distribution on the chromosome and tandem duplication analysis of $M Y B$ genes}

\section{in jujube}

The genome location of each $Z j M Y B$ member and the length of each chromosome of jujube was obtained from the Chinese jujube database (Liu et al., 2014). Based on the above information, the $Z j M Y B$ genes were mapped to corresponding locations on the twelve chromosomes using the circle gene viewer package of TBtools (Krzywinski et al., 2009; Chen et al., 2018). Tandem duplication analysis of ZjMYB genes was performed using the MCScanX tool (Wang et al., 2012).

\section{Analysis of $Z j M Y B$ gene expression from RNA-Seq data}

RNA-Seq reads were obtained with an Illumina HiSeq 2000. The fragments per kilobase of exon per million mapped reads (FPKM) values were calculated based on RNA-Seq reads. The heatmap 
was generated with TBTOOLS software (Chen et al., 2018; Liu et al., 2017); the color scale shown represents FPKM counts, and the ratios were $\log 2$ transformed. To confirm the transcriptome data, we chose $10 M Y B$ genes and quantified them using quantitative real-time PCR experiments. Total RNA was extracted using the Plant RNA Kit (TaKaRa Biotechnology Co. Ltd., Dalian, China). DNA-free RNA was used for synthesis of the first strand of cDNA by using the Prime Script II 1st Strand cDNA Synthesis Kit (TaKaRa Biotechnology Co. Ltd., Dalian, China) per the manufacturer's recommendations. Quantitative RT-PCR was carried out with the Rotor-Gene Qreal-time PCR system (Qiagen, Germany) instrument using Fast Super EvaGreen qPCR Master Mix (US Everbright Inc., Suzhou, China). The gene-specific primers of the $10 \mathrm{ZjMYB}$ genes were designed using Primer Premier 5.0 software (Lalitha, 2000), and the specific primer pairs are listed in Table S1. The UBQ gene of jujube was used as an internal control. Each reaction contained 10 $\mu \mathrm{L}$ of Fast Super EvaGreen qPCR Master Mix (US Everbright Inc., Suzhou, China), $0.4 \mu \mathrm{L}$ of each primer, $2 \mu \mathrm{L}$ of cDNA, and $7.2 \mu \mathrm{L}$ of $\mathrm{H}_{2} \mathrm{O}$ for a final volume of $20 \mu \mathrm{l}$ according to the manufacturer's instructions. The reaction was carried out as follows: $95^{\circ} \mathrm{Cfor} 2 \mathrm{~min}$, followed by 40 cycles of $95^{\circ} \mathrm{C}$ for $5 \mathrm{~s}, 60^{\circ} \mathrm{C}$ for $5 \mathrm{~s}, 72^{\circ} \mathrm{C}$ for $25 \mathrm{~s}$. Each reaction was performed using threestep amplification with three technical replicates, and the data from qRT-PCR amplification were analyzed using the $2^{-\Delta \Delta C T}$ method (Livak \& Schmittgen, 2001).

\section{Total flavonoid determination}

The determined total flavonoid content was based on the colorimetric assay method at $510 \mathrm{~nm}$, with slight modifications (Kou et al., 2015; Yu et al., 2012). Briefly, different concentrations (8, $16,20,32,48,64$, and $80 \mathrm{mg} / \mathrm{L}$ ) of a standard solution of rutin or extract was mixed with $0.3 \mathrm{~mL}$ of $5 \% \mathrm{NaNO}_{2}$. The solution was left to stand for $6 \mathrm{~min}$, and then $0.3 \mathrm{~mL}$ of $10 \% \mathrm{Al}\left(\mathrm{NO}_{3}\right)_{3}$ was added. After 6 min, $4 \mathrm{~mL}$ of $4 \% \mathrm{NaOH}$ was added. Then, the mixture was made to $10 \mathrm{~mL}$ with distilled water. The absorbance of the final mixture was measured at $510 \mathrm{~nm}$ against a prepared blank using a spectrophotometer. The total flavonoid content was analyzed using OriginPro 8.5 
210 software.

\section{Correlation analysis}

213 We performed a correlation analysis of the original FPKM counts to determine the expression

214 levels of MYB superfamily genes with the total flavonoid content. Pearson correlation analysis 215 was performed using the corr.test function of R software, and the coroplot software package of R 216 was used for significance tests and graphing. $\mathrm{P}<0.05$ was considered significant.

\section{$Z j M Y B$ gene synteny analysis}

219

220

221

222

223

224

225

226

227

228

229

230

231

232

233

234

Synteny analysis of ZjMYB genes between jujube and two plant species (peach and Arabidopsis) was performed using the MCScanX tool (Wang et al., 2012). A syntenic analysis map was constructed using TBtools software (Chen et al., 2018; Liu et al., 2017). The protein sequences and the genome location of each MYB gene and the length of each chromosome of Arabidopsis were downloaded from the TAIR database (http://www.arabidopsis.org/) (Swarbreck et al., 2008). Peach protein sequences and the genome location of each MYB gene and the length of each chromosome were downloaded from the phytozome database (https://phytozome.jgi.doe.gov/) (Zhang \& Ma et al., 2018).

\section{Results}

\section{Identification of jujube $M Y B$ genes and analysis of their protein} physicochemical properties

A total of 171 ZjMYB genes with typical MYB or MYB-like domains were selected from the jujube genome database on the basis of the HMM profile of the MYB domain. The corresponding chromosomal locations were provisionally ordered by name such that the $M Y B$ genes were named ZjMYB1 through ZjMYB171. The geneIDs, gene lengths, and physiological and biochemical 
235

236

237

238

239

240

241

242

243

244

245

246

247

248

249

250

251

252

253

254

255

256

257

258

259

260

261

characteristics of the ZjMYB genes are listed in Table S2. The lengths of the coding sequences (CDSs) of the ZjMYB genes ranged from 276 to $3645 \mathrm{bp}$. The lengths of the protein sequences of $Z j M Y B$ genes ranged from 91 to 1214 amino acids. The isoelectric point (pI) values for $Z j M Y B$ proteins ranged from $4.78(Z j M Y B 108)$ to 10.39 (ZjMYB145), with an average of 7.21 , which is similar to those for MYB proteins in sesame (Sesamum indicum L.) and peach (Mmadi et al., 2017; Zhang \& Ma et al., 2018). The molecular weight (Mw) ranged from 10558.01 Da (ZjMYB74) to 136172.41 Da (ZjMYB23), with an average of 42499.15 Da. The GRAVY (grand average of hydropathy) average value was -0.74 , which is similar to that for peach MYB proteins (Zhang \& Ma et al., 2018). The most likely homologous gene of each jujube MYB gene in Arabidopsis was obtained from the BLAST tool in the TAIR database (Table S2).

\section{Phylogenetic trees and group classification of $Z j M Y B$ proteins}

This study identified $171 M Y B$ genes in jujube. As shown in Figures 1 and 2, we found 58 MYBrelated proteins, 99 R2R3-MYB proteins, 4 R1R2R3-MYB proteins, 1 4R-MYB protein, and 9 atypical MYB proteins. R2R3-MYB proteins accounted for $58 \%$ of the $Z j M Y B$ proteins as the largest subfamily, and 4R-MYB subfamily proteins accounted for $0.6 \%$ as the smallest subfamily. MYB-related proteins accounted for $40 \%$ of $Z j M Y B$ proteins as the second largest subfamily. The first phylogenetic tree (Fig. 1A) comprised 171 ZjMYB superfamily genes and 126 R2R3MYB genes, 5 R1R2R3-MYB genes, and 1 4R-MYB gene of Arabidopsis. Fifty-eight MYBrelated proteins and 9 atypical MYB proteins of the ZjMYB superfamily were clustered alone. Then, we used those genes with 60 MYB-related and 6 atypical MYB proteins of Arabidopsis to construct the second phylogenetic tree (Fig. 2A). As shown in Figure 1A, 1 jujube 4R-MYB protein (ZjMYB62) was clustered into the 4R-MYB protein of Arabidopsis, 4 jujube R1R2R3MYB proteins were clustered into 5 R1R2R3-MYB proteins of Arabidopsis, and 99 jujube R2R3MYB proteins were clustered into 126 R2R3-MYB proteins of Arabidopsis. These 99 R2R3-MYB genes of jujube were divided into 35 groups, $\mathrm{C} 1$ to $\mathrm{C} 35$, based on the topology of the tree and the classification of the MYB superfamily in Arabidopsis, peach and pear (Pyrus bretschneideri) 
262 (Dubos et al., 2010; Zhang \& Ma et al., 2018; Li X et al., 2016). The number of members in each 263 group ranged from 1 to 7 in jujube. C10 had 7 members and was the largest group.

264 In the second phylogenetic tree (Fig. 2A), 58 MYB-related (1R-MYB) proteins were divided into 2656 groups based on the topology of the tree and their classifications in Arabidopsis and peach (Chen 266 et al., 2006; Zhang \& Ma et al., 2018). CPC-like (3 members), TBP-like (11 members), I-box267 binding-like (4 members), R-R-type (5 members), CCA1-like (13 members), and Chinese jujube268 specific (22 members) groups were present. TBP-like was the second largest group among the six groups of the MYB-related subfamily, as in peach (Zhang \& Ma et al., 2018). The 22 members did not cluster with any Arabidopsis proteins. However, this result has been shown in previous studies in peach (Zhang \& Ma et al., 2018) and sweet orange (Hou et al., 2014), and the authors included those genes in the MYB-related (1RMYB) subfamily. The author of the peach study reported that those genes might have fruit-related functions and were either not needed in Arabidopsis or were obtained after divergence from the last common ancestor (Zhang \& Ma et al., 2018). This study further contributes to this speculation as the function of those genes remains unknown. Nine atypical MYB proteins were clustered in 4 branches in the phylogenetic tree.

\section{$Z j M Y B$ gene structure and protein motif analysis}

280

The MEME program predicted 15 conserved motifs of the MYB proteins of jujube, which appeared in R2R3-MYB and R1R2R3-MYB (Table S3 and Fig. 1B), MYB-related and atypical MYB (Table S4 and Fig. 2B), and 4R-MYB (Table S5). The gene exon/intron structures were analyzed in R2R3-MYB with R1R2R3-MYB (Table S3 and Fig. 1C), MYB-related with atypical MYB (Table S4 and Fig. 2C), and 4R-MYB.

R2R3-MYB and R1R2R3-MYB had 3 identical highly conserved motifs (Fig. 1B), motif 3, motif 2, and motif 1. Seventy-eight of the 99 R2R3-MYB proteins had 4 identical highly conserved motifs, motif 3, motif 4, motif 2 and motif 1 . As the sequence logos of the R2R3-MYB protein repeats show (Fig. 3), motif 3, motif 4, and the front part of motif 2 constitute the R2 repeat in 
289 jujube and include a highly conserved separated triplet of Tryptophan (W) to maintain the HTH 290 structure. This result was consistent with those of previous studies (Zhang \& Ma et al., 2018; Hou 291 et al., 2014; Chen et al., 2006) showing that the back parts of motif 2 and motif 1 constituted the 292 R3 repeat. In this study, the first Tryptophan (W) of the R3 repeat in jujube was replaced by 293 Leucinol hydrochloride (L), Isoleucine (I), Phenylalanine (F), Tyrosine (Y) and Methionine (M) 294 (the back part of motif 2 in Fig. 3). The second and third Tryptophan residues were conserved in 295 most R3 repeats in this study; only 1 (ZjMYB170) occurred in group C34 (2 members), and the third Tryptophan (W) residue was replaced by Phenylalanine (F) (the last amino acid of motif 1 in Fig. 3). Consistent with previous research, the R2 repeat also had the following highly conserved groups of EED residues (motif 3): glutamic acid (E)-glutamic acid (E)-aspartic acid (D) and EEE residues (motif 2) (Zhang \& Ma et al., 2018; Li X et al., 2016). In jujube, the remaining residues were highly conserved in the R2 repeat; the eighth and sixteenth residues behind the first Tryptophan (W) were leucine (L) and glycine (G), and the eighth $(\mathrm{G})$ and ninth $(\mathrm{L})$ residues were behind the second Tryptophan (W). In front of the third Tryptophan (W) in the R2 repeats, the following 9 consecutive highly conserved residues were observed: arginine (R), cysteine (C), Glycine $(\mathrm{G})$, Lysine $(\mathrm{K})$, Serine $(\mathrm{S})$, cysteine $(\mathrm{C})$, arginine $(\mathrm{R})$, leucine $(\mathrm{L})$, and arginine $(\mathrm{R})$. This characteristic was also found in other plant species, such as peach (Zhang \& Ma et al., 2018) and Chinese white pear (Li X et al., 2016). Although the authors did not mention this characteristic in their articles, we found it by reanalyzing the data they presented. Other R2R3-MYB proteins (21 members) did not contain motif 4, and they were divided into 8 groups, including 1 in group C7 (3 members) and all members in group C29 to C35. Fifty-seven of the 99 R2R3-MYB proteins included motif 3, motif 4, motif 2 and motif 1 and also included motif 6. Although ZjMYB113 did not have motif 5 and ZjMYB42 did not have motif 6, groups C1, C2, C3, C9, and C7 had motifs 6, 3, 4, 2, 1 and 5. Groups C16 and C4 had motifs 6, 3, 4, 2, 1, 5 and 8. Motif 8 had highly conserved Tryptophan (W) residues, and the function of this $\mathrm{W}$ requires further research. The members of groups C11, C32, C30 and C34 had unique motifs, including 11, 12, 13 and 14 in jujube R2R3MYB proteins. 
316 Four R1R2R3-MYB proteins contained motifs 9, 3, 10, 2, 1 and 7. As the sequence logos show

317 (Fig. 4), motif 9 constituted the R1 repeat; motifs 3, 10 and 2 constituted the R2 repeat; and motifs

3181 and 7 constituted the R3 repeat. The R2 repeat differed between R2R2-MYB and R1R2R3-MYB.

319 Motifs 3, 4 and 2 constituted the R2 repeat of R2R3-MYB.

320 The coding sequences of all R2R3-MYBs were disrupted by introns (Fig. 1C), except for group

321 32. Most coding sequences had three exons and two introns, and all of these exons included 2

322 short exons and 1 relatively long exon, similar to those in peach (Zhang \& Ma et al., 2018). All

323 group 32 members had no introns and only 1 exon. However, these members had green regions

324 upstream and downstream of the front and back end of the sequences, which was consistent with

325 those in peach. Two members of group C34 had a maximum of 11 introns in the R2R3-MYB

326 subfamily. The members of groups 18 and 19 had 2 exons and 1 intron. The gene length, exon

327

328

329

330

331

332

333

334

335

336

337

338

339

340

341

342

number and exon/intron structure within the same group of R2R3-MYB were similar, which

supported the classification of R2R3-MYB subfamilies. R1R2R3-MYB introns ranged from 6 to 12, and ZjMYB134 had a maximal number of 12 introns.

Although comprehensive analyses of the motifs of R2R3-MYB proteins haves been performed in previous studies, a similar comprehensive motif analysis for the MYB-related subfamily is lacking. In this study, 15 motifs of 58 MYB-related proteins and 9 atypical MYB proteins are shown in Figure 2B The groups contained a highly conserved motif 2 except the Chinese jujube-specific group. The specific group consisted of at least conserved motifs 1 and 2, which together comprised the $\mathrm{R} 1$ repeat. The members of the R-R-type group had motifs 2, 5, 2 and 3 in that order and contained 2 repeats. Motifs 2 and 5 comprised the first repeat, and motifs 2 and 3 comprised the second repeat. The CCA1-like group was divided into 2 parts consisting of at least conserved motifs 2 and 3, which together comprised the R1 repeat. All CCA1-like members contained the conserved motif SHAQK with motif 3 of the MYB repeat, consistent with previous studies in Arabidopsis (Chen et al., 2006). Nine members of the second part of the CCA1-like group (12 members) also contained motif 8. Four members of the I-box-like group had motifs 2 and 5, which together constituted the R1 repeat of the I-box-like group. The lengths of all I-box-binding-like 
343 protein sequences were short and similar. The 11 members of TBP-like had motifs 2 and 7 except 344 for ZjMYB29. Five members of 9 atypical MYB proteins had only motif 2, and other atypical MYB 345 proteins had motifs 2 and 2 in that order, and they comprised an imperfect R1 repeat.

346 To clearly display the repeats of the 7 different groups in MYB-related proteins, we used the 347 MEME program to define the motifs of 7 groups with highly conserved motifs (Fig. 5 and Table 348 S6). The motifs in the sequence logos of R-R-type repeats had higher similarity than did those in 349 Arabidopsis and peach (Chen et al., 2006; Zhang \& Ma et al., 2018). The CCA1-like subfamily is 350 composed of two main clades and is the same in Arabidopsis (Chen et al., 2006). The primary 351 structure of clade I repeat DNA-binding domains was [-W-(X 19)-W-], and the repeat of clade 352 II was [-W-(X 18)-W-]. The primary structure of the TBP-like repeat DNA-binding domains was [-W-(X 19)-W-]. Most R1 repeats of MYB-related proteins contained highly conserved groups of EED or EEE residues in jujube.

355 The coding sequences of the MYB-related subfamily were also disrupted by introns (Fig. 2C).

356 The R-R-type and I-box-binding-like genes had 2 exons and 1 intron except for ZjMYB33 of the 357 R-R type, which was similar to groups 18 and 19 of the R2R3-MYB subfamily. Most Chinese 358 jujube-specific genes had 4 and 5 introns except for 3 genes that containing 6 introns and 1 that 359 had 3 introns. On average, the CCA1-like group was disrupted by 4.8 introns, the CPC-like 360 group had 13 introns, the atypical MYB group had 4.1 introns, and the TBP-like group had 4.8 361 introns.

362 We used the MEME program to define the motifs of 4R-MYB (ZjMYB62) and Arabidopsis 363 (AT3G18100.1). Their motifs were very similar, and they all included highly conserved motifs 1 364 to 15. Seven groups had highly conserved motifs (Fig. 6A and Table S5), indicating that their 365 sequences were similar. These groups contained 4 MYB DNA-binding domains. The jujube $4 R-$ 366 $M Y B$ gene had 9 introns (Fig. 6B), and the first intron was the longest. 


\section{9 in jujube}

370 Jujube has twelve chromosomes (Zj01 to Zj12) (Fig. 7), and 142 of $171 \mathrm{ZjMYBs}$ unevenly 371 distributed on chromosomes 1 to 12 of the jujube genome (Table S2 and Fig. 7). From Figure 7, 372 we can find the higher density of the chromosome genes, and the number of ZjMYBs was also 373 relatively large on each chromosome. $\mathrm{Zj01}$ is the largest chromosome and had the most ZjMYBs. 374 Three tandem duplicated pairs of jujube MYB genes were found on chromosome 2 (ZjMYB25 with 375 ZjMYB26), chromosome 5 (ZjMYB64 with ZjMYB65) and chromosome 11 (ZjMYB123 and 376 ZjMYB124).

\section{Analysis of $Z j M Y B$ gene expression from RNA-Seq data}

The raw transcriptome sequences from RNA-Seq have been deposited into the NCBI sequence read archive (SRA) under accessions SRP162927. As shown in Table S7, a total of 46,464,880 $50,619,884$ raw reads were generated from the fifteen libraries. After filtration, a total of 46,244,076 - 50,550,982 clean reads were obtained from the fifteen libraries with an average Raw Q30 and Clean Q30 base rate of nearly 94\%. The overall quality of the sequence data was suitable for further analysis. As revealed by jujube RNA-Seq (Fig. 8A and 8B), the expression levels of different genes at different developmental stages varied. TBtools software yielded a clearer picture of the trend of expression levels of different genes in different stages; the row was also $\log 2$ transformed. The reliability of the RNA-Seq data was further validated through real-time quantitative PCR (RT-qPCR) experiments which were carried out on 10 selected MYB genes at five stages (Fig. 8C).

Although extensive analysis of the expression levels of R2R3-MYB genes has been performed (Kranz, et al., 1998; Stracke, Werber \& Weisshaar, 2001; Jia, Clegg \& Jiang, 2004), a similar analysis of 1R-MYB (MYB-related) genes is limited. Interestingly, based on the original FPKM counts in the rectangles, the expression levels of most MYB-related genes (Fig. 8B) were relatively higher than those of R2R3-MYB, R1R2R3-MYB and 4R-MYB genes (Fig. 8A) in jujube fruit. Based on the expression profiles of the ZjMYB family, the R2R3-MYB, R1R2R3-MYB and 4R- 
396

397

398

399

400

401

402

403

404

405

406

407

408

409

410

411

412

413

414

415

416

417

418

419

420

421

422

MYB subfamilies can be divided into 9 subgroups, A1-A9 (Fig. 8A), and the MYB-related subfamily can be divided into 8 subgroups, B1 to B8 (Fig. 8B).

Interestingly, 4 genes (ZjMYB126, ZjMYB7, ZjMYB10 and ZjMYB72) in group B5 and 2 genes (ZjMYB92 and ZjMYB127) in group A7 similar changes in their gene expression levels, and all these genes showed a trend from high to low in Y to F. In contrast, the ZjMYB34 gene in group A1 and 3 genes (ZjMYB23, ZjMYB41 and ZjMYB67) in group B3 had similar gene expression changes from low to high in $\mathrm{Y}$ to FR. According to previous studies, the content of total flavonoids, phenolics and proanthocyanidins decrease as ripening progresses (Wu et al., 2012). Therefore, we predicted that the 10 genes of groups B3, B5, A1 and A7 may possess the function of activating flavonoids and proanthocyanidin biosynthesis.

In A7, most genes displayed high expression at stages $\mathrm{Y}$ and $\mathrm{EN}$ and had lower expression levels and even no expression in the other stages. Based on this finding, we can infer that the genes in group A7 probably participate in important regulatory functions at the early stages of jujube fruit development. In contrast, group A2 had relatively high expression levels at the late stages (WM, HR and FR). Compared with the genes in other groups, most genes in groups A1 (except for ZjMYB34), A3 (except for ZjMYB99), A4 (except for ZjMYB88), A6, A8 (except for ZjMYB136), A9 (except for ZjMYB48), B4 (except for ZjMYB63) and B8 had lower expression levels, as well as no expression. The gene expression trend from Y to FR in group B3 and ZjMYB34 in group A1 was from low to high. The expression level of group A2 increased during the early stages and decreased in the middle stage of jujube fruit development. These results indicated that $M Y B$ genes may participate in different regulatory mechanisms during jujube fruit development.

\section{Total flavonoid determination}

Consistent with previous studies, the total flavonoid content of jujube decreased with jujube fruit ripening (Wu et al., 2012) (Fig. 9). At the FR stage, the content $(0.44 \pm 0.03 \mathrm{mg} \mathrm{RE} / \mathrm{g} \mathrm{FW})$ was similar to that in Z. jujube cv. Hupingzao $(0.47 \pm 0.06 \mathrm{mg} \mathrm{RE} / \mathrm{g} \mathrm{FW})$ (Kou et al., 2015). The total flavonoid content was highest in the $\mathrm{Y}$ stage and reached $1.23 \pm 0.03 \mathrm{mg} \mathrm{RE} / \mathrm{g} \mathrm{FW}$. 


\section{Correlation analysis}

425

426

427

428

429

430

431

432

433

434

435

436

437

438

439

440

441

442

443

444

445

446

447

448

To further explore the associations between the original FPKM counts of transcriptome data of 93 ZjMYB genes (containing 42 MYB-related, 7 atypical MYB, 40 R2R3-MYB, 3 R1R2R3-MYB and 1 4R-MYB genes) and total flavonoid contents, we performed a correlation analysis using $\mathrm{R}$ software (Fig. 10). Fifty-six MYB genes (23 MYB-related, 4 atypical MYB, 26 R2R3-MYB, 2 R1R2R3-MYB and 1 4R-MYB genes) presented significant $(<0.05)$ correlations.

Among these genes, 7 MYB-related (ZjMYB23, ZjMYB41, ZjMYB67, ZjMYB109, ZjMYB111, ZjMYB121 and ZjMYB139), 1 atypical MYB (ZjMYB110), eight R2R3-MYB (ZjMYB4, ZjMYB9, ZjMYB34, ZjMYB44, ZjMYB45, ZjMYB107, ZjMYB135 and ZjMYB142) and one 4R-MYB gene (ZjMYB62) had a negative correlation with the total flavonoid content. Sixteen MYB-related genes (ZjMYB2, ZjMYB7, ZjMYB10, ZjMYB11, ZjMYB18, ZjMYB63, ZjMYB72, ZjMYB76, ZjMYB95, ZjMYB98, ZjMYB100, ZjMYB117, ZjMYB119, ZjMYB126, ZjMYB132 and ZjMYB133), 3 atypical MYB genes (ZjMYB84, ZjMYB122 and ZjMYB125), eighteen R2R3-MYB genes (ZjMYB5, ZjMYB31, ZjMYB32, ZjMYB43, ZjMYB49, ZjMYB51, ZjMYB59, ZjMYB61, ZjMYB78, ZjMYB81, ZjMYB92,ZjMYB102, ZjMYB108, ZjMYB114,ZjMYB127, ZjMYB136,ZjMYB140 and ZjMYB141) and two R1R2R3-MYB (ZjMYB79 and ZjMYB134) genes were positively correlated with total flavonoid contents.

$Z j M Y B$ gene synteny analysis

Arabidopsis is the most important model plant, and many MYB genes in Arabidopsis have been functionally well characterized. To further determine the phylogenetic mechanisms of the jujube MYB superfamily, we constructed a map based on a syntenic analysis of MYB genes between jujube with other species, including plants from the Rosaceae (peach) and Brassicaceae (Arabidopsis) as well as the paralogous MYB genes in jujube (Fig. 11) to predict the function of $Z j M Y B s$ and analyze the phylogenetic mechanisms of $M Y B$ genes. This process only highlighted 
449 the $M Y B$ genes for each species. To find more homologous $M Y B$ gene pairs between jujube and 450 other species, their common gene pairs were all located using syntenic blocks possessing fewer 451 than 5 homologous gene pairs when we visualized the results using TBtools software.

452 The 5 pairs of paralogous $M Y B$ genes within jujube were thus composed of 9 jujube $M Y B$ genes 453 (Fig. 11 (red line) and Table S8). Fifty-seven pairs of orthologous $M Y B$ genes in jujube and 454 Arabidopsis were identified among the 171 ZjMYBs and 198 AtMYBs (Fig. 11 (green line) and 455 Table S8). Thirty-seven pairs of orthologous $M Y B$ genes in jujube and peach were identified 456 among the $171 \mathrm{ZjMYBs}$ and 256 PpMYBs (Fig. 11 (blue line) and Table S8). Sixteen and 7 MYB 457 genes of jujube showed homology with multiple $M Y B$ genes from various locations on different 458 chromosomes in Arabidopsis and peach, respectively. Fourteen ZjMYB genes of jujube showed 459 the same homology to the MYB genes of Arabidopsis and peach.

\section{Discussion}

A total of $171 Z j M Y B$ genes were identified. Differences in the number of $M Y B$ genes among diverse species have been studied. For instance, 256 MYB genes were found in peach (Zhang \& Ma et al., 2018), 177 in sweet orange (Citrus sinensis) (Hou et al., 2014) and 125 in physic nut (Zhou et al., 2015). According to previous studies, the MYB family is the largest TF family in jujube (Liu et al., 2017; Song et al., 2017; Shao et al., 2017; Zhang \& Ma et al., 2017), consistent with previous reports in Arabidopsis (Riechmann et al., 2000). The ZjMYB genes can be divided into five subfamilies, including 58 MYB-related proteins, 99 R2R3-MYB proteins, 4 R1R2R3MYB proteins, 1 4R-MYB protein, and 9 atypical MYB proteins. The R2R3-MYB proteins make up the largest subfamily, consistent with previous reports in peach and Arabidopsis. Similar to jujube, a total of 128 R2R3-MYB proteins were identified in peach (Zhang \& Ma et al., 2018), and 126 were found in Arabidopsis (Chen et al., 2006). Additionally, R2R3-MYB is the largest subfamily of the MYB superfamily. However, this finding is inconsistent with the MYB-related subfamily having the largest number of MYB superfamily genes in sesame (Mmadi et al., 2017). 
476 These differences are most likely associated with the differences in the evolution of these plants.

477 In this study, we identified $14 \mathrm{R}-\mathrm{MYB}$ protein, which is consistent with previous studies of 478 Arabidopsis (Dubos et al., 2010), peach (Zhang \& Ma et al., 2018) and pear (Li X et al., 2016).

479 However, the results of this study are inconsistent with those of previous studies reporting 4 4R480 MYB proteins in Chinese cabbage (Brassica rapa ssp pekinensis) (Saha et al., 2016), 2 4R-MYB 481 proteins in upland cotton (Gossypium hirsutum L.) (Salih et al., 2016), and 0 4R-MYB proteins in 482 sesame (Mmadi et al., 2017). In this study, we identified 4 R1R2R3-MYB proteins, which is 483 consistent with previous studies on peach (Zhang \& Ma et al., 2018) and tomato (Li ZJ et al., 2016), but these results are inconsistent with other previous studies reporting 11 R1R2R3-MYB proteins in Chinese cabbage (Saha et al., 2016), 5 R1R2R3-MYB proteins in sesame (Mmadi et al., 2017) and 15 3R-MYB proteins in upland cotton (Salih et al., 2016). These differences are most likely associated with differences in the evolution of these plants. In previous studies, the first Tryptophan (W) residue of the R3 repeat was replaced by Leucinol hydrochloride (L), Isoleucine (I) and Phenylalanine (F) (Ogata eta al., 1994; Du et al., 2012b). However, in this study, the first Tryptophan (W) of the R3 repeat in jujube was replaced by Tyrosine (Y) and Methionine (M) in addition to the above findings (the back part of motif 2 in Fig. 3); there were 2 (ZjMYB53 and ZjMYB54) in group C5 (3 members) and 1 (ZjMYB12) in group C12 (1 member). The second and third Tryptophan residues in this study were conserved in most R3 repeats, with 1 (ZjMYB170) occurring in group C34 (2 members), and the third Tryptophan (W) residue was replaced by Phenylalanine (F) (the last amino acid of motif 1 in Fig. 3). This finding might be attributed to the loss of residues during jujube evolution or a change in response to changes in the environment, further illustrating the diversity of the MYB domain (Du et al., 2012a). Consistent with previous research, the R2 repeat of R2R3-MYB genes also had highly conserved groups of EED and EEE residues (Zhang \& Ma et al., 2018; Li X et al., 2016) (Fig. 3). In jujube, the other residues were highly conserved in the R2 repeat; the eighth and sixteenth residues behind the first Tryptophan (W) were Leucine (L) and Glycine (G), and the eighth (G) and ninth (L) residues were behind the second Tryptophan (W). 
503

504

505

506

507

508

509

510

511

512

513

514

515

516

517

518

519

520

521

522

523

524

525

526

527

528

529

Although many comprehensive analyses of the motif for R2R3-MYB proteins have been performed in previous studies, a similar comprehensive analysis of the motif for the MYB-related subfamily is lacking. The 58 MYB-related (1R-MYB) proteins were divided into 6 groups, including the CPC-like, TBP-like, I-box-binding-like, R-R-type, CCA1-like, and Chinese jujubespecific groups (Fig. 2). Interestingly, the MYB repeats of CCA1-like proteins are closely related to R-R type MYB repeats (R-R(B)), and I-box-like protein MYB repeats are closely related to RR-type MYB repeats (R-R(A)) (Fig. 5). We speculate that CCA1-like proteins and I-box-like proteins may be induced by R-R-type gene loss events.

The R2 repeat had highly conserved groups of EED and EEE residues in other plant species, such as peach (Zhang \& Ma et al., 2018), pear (Li X et al., 2016) and tomato (Li ZJ et al., 2016). Interestingly, most R1 repeats of MYB-related proteins also included highly conserved groups of EED or EEE residues in jujube (Fig. 5).

The exon numbers of 171 ZjMYB genes ranged from 1 to 20 (Fig. 1C and Fig. 2C), indicating the loss and gain of ZjMYB exons during gene evolution, which may account for the functional diversity of the ZjMYB subgroup. As suggested by previous studies, the conserved motifs and intron/exon structures in each subgroup probably play important roles in each group's specific functions (Zhang \& Ma et al., 2018; Li X et al., 2016).

From the Figure 7, we can see that more MYB genes are located at the two ends of the chromosome than on the middle of the chromosome, and similar patterns were also reported in peach (Zhang \& Ma et al., 2018) and sweet orange (Hou et al., 2014). Despite the lack of description in the article, we can see from a previous paper that the MYB genes of Arabidopsis had similar distribution patterns on chromosome 1 and chromosome 5 (Katiyar et al., 2012).

The expression of most MYB-related genes (Fig. 8B) was relatively higher than that of R2R3MYB, R1R2R3-MYB and 4R-MYB genes (Fig. 8A) in jujube fruit. Thus, we speculated that MYB-related genes probably play a greater role in fruit-related functions. Many of the functions of R2R3-MYB genes have been studied, but the function of MYB-related genes remains unknown. Therefore, we suggest further functional studies of MYB-related genes in the future. 
530 Consistent with previous studies, the total flavonoid content of jujube decreased with jujube fruit 531 ripening (Wu et al., 2012) (Fig. 9). According to previous studies, the MYB TF of Arabidopsis is 532 involved in many secondary metabolic processes, such as the flavonoid metabolic pathway, 533 glucosinolate biosynthesis, and anthocyanin biosynthesis (Stracke et al., 2010; Bhatia et al., 2018; 534 Wei et al., 2015). For example, the MdMYBPA1 TF of red-fleshed apple responded to low 535 temperatures by redirecting the flavonoid biosynthetic pathway from proanthocyanidin to 536 anthocyanin production (Liu et al., 2015). The MYB superfamily in other plants is also involved 537 in flavonoid/phenylpropanoid metabolism (Puche et al., 2014; Ampomah -Dwamena et al., 2018; 538 Wang \& Qu et al., 2018). By correlation analysis between the original FPKM counts of 539 transcriptome data from 93 ZjMYB genes (Fig. 10), 56 MYB genes presented a significant 540 correlation.

541 Among the orthologous pairs (Fig. 11) (ZjMYB108 with AtMYB12 and AtMYB11; ZjMYB46 with 542 AtMYB21, AtMYB24 and AtMYB57; ZjMYB88 with AtMYB15; ZjMYB69 with AtMYB44; ZjMYB99 543 with AtMYB44 and AtMYB77; ZjMYB65 with AtMYB28; ZjMYB107 with AtMYB2; ZjMYB118 544 with AtMYB62;) in jujube and Arabidopsis, AtMYB12 (AT2G47460) was expressed at low 545 temperature in a light-dependent manner, and overexpression of the AtMYB12 gene enhanced 546 flavonoid accumulation (Bhatia et al., 2018). AtMYB11 and AtMYB12 had a high degree of 547 functional similarity and controlled flavanol biosynthesis (Stracke et al., 2010). We speculate that 548 the ZjMYB108 gene may have the same function. In the correlation analysis results (Fig. 10), 549 ZjMYB 108 gene expression has a positive correlation with flavonoid content; therefore, we further 550 speculate that $Z j M Y B 108$ is an important transcription factor involved in the flavonoid metabolic 551 pathway. AtMYB21 (AT3G27810) is involved in the light signaling pathway (Shin et al., 2002). 552 GA (gibberellin) promotes JA (jasmonate) biosynthesis to control the expression of AtMYB21 553 (AT3G27810), AtMYB24 (AT5G40350), and AtMYB57 (AT3G01530) (Cheng et al., 2009). 554 Transgenic Arabidopsis expressing AtMYB15 improved tolerance to cold (Agarwal et al., 2006) 555 and drought stress (Ding et al., 2008). AtMYB44 (AT5G67300) was rapidly induced by MeJA 556 (methyl jasmonate) in Arabidopsis. Transgenic Arabidopsis overexpressing the AtMYB44 gene 
557

558

559

560

561

562

563

564

565

566

567

568

569

570

571

572

573

574

575

576

577

578

579

580

581

582

583

was more sensitive to ABA and exhibited a markedly increased tolerance to salt and drought stress compared to wild-type plants (Jung et al., 2008). MYB77 (At3G50060) regulates auxin signaling processes and auxin concentrations (Shin et al., 2007). AtMYB28 (AT5G61420) is involved in aliphatic glucosinolate biosynthesis (Gigolashvili et al., 2007). Under drought stress in plants, the AtMYB2 (AT2G47190) protein can function as a transcriptional activator in ABA-inducible gene expression (Abe et al., 2003). AtMYB62 (AT1G68320) regulates phosphate starvation responses and gibberellic acid biosynthesis (Devaiah et al., 2009). The genes of the MYB orthologous pairs of jujube with Arabidopsis may have the same functions. We predict that the ZjMYBs are involved in flavonoid/phenylpropanoid metabolism, the light signaling pathway, auxin signal transduction, and responses to the various abiotic stresses (cold, drought and salt stresses). Fourteen ZjMYB genes of jujube had the same homology in the MYB genes of Arabidopsis and peach, indicating that the $14 M Y B$ genes as well as the orthologous pairs with those 14 genes probably existed before the ancestral divergence of the MYB superfamily.

\section{Conclusions}

In this study, we performed the first genome-wide detailed analysis of jujube $M Y B$ superfamily genes, including 171 MYB genes (containing 58 MYB-related genes, 99 R2R3-MYB genes, 4 R1R2R3-MYB genes, 1 4R-MYB gene, and 9 atypical MYB genes). These 99 R2R3-MYB genes in jujube were divided into 35 groups, $\mathrm{C} 1$ to $\mathrm{C} 35$, and $58 \mathrm{MYB}-$ related genes were divided into the following groups: the R-R-type, CCA1-like, I-box-binding-like, TBP-like, CPC-like and Chinese jujube-specific groups. The members of the $Z j M Y B$ gene superfamily in jujube were well supported by additional highly conserved motifs and exon/intron structures. Most R1 repeats of MYB-related proteins that also contained R2 repeats included highly conserved groups of EED and EEE residues in jujube. The composition of the motifs of the 4R-MYB protein in jujube was similar to that in Arabidopsis. The exon numbers of ZjMYB genes ranged from 1 to 20, indicating loss and gain of $Z j M Y B$ exons during gene evolution. Three tandem duplicated gene pairs were found on twelve chromosomes of jujube. The analysis of ZjMYB gene expression indicated that 
584

585

the different genes had varying expression levels across developmental stages. Interestingly, in this study, the expression of most MYB-related gene expression was higher than that of R2R3MYB, R1R2R3-MYB and 4R-MYB genes. In previous studies, the R2R3-MYB subfamily has received more attention. However, in the present study, analysis of the expression of $126 \mathrm{ZjMYB}$ genes revealed that MYB-related genes played important roles in jujube development and participated in fruit-related biological processes. The total flavonoid content of jujube decreased with increasing ripening of jujube fruits. Ninety-three expressed genes were identified from the RNA-sequencing data of jujube fruit, and 56 ZjMYB members presented a significant correlation with the total flavonoid contents by correlation analysis. Five pairs of paralogous $M Y B$ genes within jujube were thus composed of 9 jujube $M Y B$ genes. Fourteen $Z j M Y B$ genes of jujube showed close homology to the $M Y B$ genes of Arabidopsis and peach, indicating that these 14 MYB genes as well as their orthologs probably existed before the ancestral divergence of the MYB superfamily. Based on a synteny analysis of $M Y B$ genes between jujube and Arabidopsis, we predict that $Z j M Y B s$ are involved in flavonoid/phenylpropanoid metabolism, the light signaling pathway, auxin signal transduction, and responses to various abiotic stresses (cold, drought and salt stresses). We also speculate that $Z j M Y B 108$ is an important transcription factor involved in the flavonoid metabolic pathway. This study provided useful information that may serve as the basis for functional analyses and cloning of ZjMYB genes. However, further studies are needed to explore the functions of the $Z j M Y B$ genes to reveal the molecular regulatory of the mechanisms of these genes in jujube fruit development.

\section{References}

Abe H, Urao T, Ito T, Seki M, Shinozaki K, Yamaguchishinozaki K. 2003. Arabidopsis AtMYC2 (bHLH) and AtMYB2 (MYB) function as transcriptional activators in abscisic acid signaling. The Plant Cell 15(1), 63-78. DOI: $10.1105 / \mathrm{tpc} .006130$

Agarwal M, Hao Y, Kapoor A, Dong CH, Fujii H, Zheng X, Zhu J. 2006. A R2R3 type MYB transcription factor is involved in the cold regulation of CBF genes and in acquired freezing tolerance. Journal of 
611

612

613

614

615

616

617

618

619

620

621

622

623

624

625

626

627

628

629

630

631

632

633

634

635

636

637

Biological Chemistry 281(49), 37636-37645. DOI: 10.1074/jbc.M605895200

Albert N W, Lewis D H, Zhang H, Schwinn KE, Jameson PE, Davies Km. 2011. Members of an R2R3-MYB transcription factor family in Petunia are developmentally and environmentally regulated to control complex floral and vegetative pigmentation patterning. Plant Journal 2011, 65(5):771-784. DOI: 10.1111/j.1365-313X.2010.04465.X

Allan AC, Hellens RP, Laing WA. 2008. MYB transcription factors that colour our fruit. Cell 13:99-102. DOI: 10.1016/j.tplants.2007.11.012

Ampomah-Dwamena C, Thrimawithana AH, Dejnoprat S, Lewis D, Espley RV, Allan AC. 2018. A kiwifruit (Actinidia deliciosa) R2R3-MYB transcription factor modulates chlorophyll and carotenoid accumulation. New Phytol. Doi: 10.1111/nph.15362

Ballesteros ML, Bolle C, Lois LM, Moore JM, Vielle-Celzada JP, Grossniklaus U, Chua NH. 2001. LAF1, a MYB transcription activator for phytochrome A signaling. Genes \& Development 15(19):2613-25. DOI: 10.1101/gad.915001

Bailey TL, Boden M, Buske FA, Frith M, Grant CE, Clementi L, Ren J, Li WW, Noble WS. 2009. MEME SUITE: tools for motif discovery and searching. Nucleic Acids Res 37(Web Server issue):W202-W208. DOI: $10.1093 /$ nar/gkp33

Bhatia C, Pandey A, Reddy-Gaddam S, U Hoecker, Trivedi PK. 2018. Low Temperature Enhanced Flavonol Synthesis Requires Light-Associated Regulatory Components in Arabidopsis thaliana. Plant and Cell Physiology. DOI: 10.1093/pcp/pcy132

Chen CJ, Xia R, Chen H, He YH. 2018. TBtools, a Toolkit for Biologists integrating various HTS-data handling tools with a user-friendly interface. BioRxiv. DOI: 10.1101/289660

Chen YH, Yang XY, He K, Liu MH, Li JG, Gao ZF, Lin ZQ, Zhang YF, Wang XX, Qiu XM, Shen YP, Li Z, Deng XH, Luo JC, Deng XW, Chen ZL, Gu HY, Qu LJ. 2006. The MYB transcription factor superfamily of Arabidopsis thaliana: expression analysis and phylogenetic comparison with the rice MYB family. Plant Molecular Biology 60:107-124. DOI:10.1007/s11103-005-2910-y

Cheng H, Song S, Xiao L, Soo H L, Cheng Z, Xie D, Peng J. 2009. Gibberellin Acts through Jasmonate to Control the Expression of MYB21, MYB24, and MYB57 to Promote Stamen Filament Growth in 
638

639

640

641

642

643

644

645

646

647

648

649

650

651

652

653

654

655

656

657

658

659

660

661

662

663

664

Arabidopsis. PLOS Genetics 5(3). DOI: 10.1371/journal.pgen.1000440

Colanero S, Perata P, Gonzali S. 2018. The atroviolacea Gene Encodes an R3-MYB Protein Repressing

Anthocyanin Synthesis in Tomato Plants. Frontiers in Plant Science 9:830. DOI: 10.3389/fpls.2018.00830

Cominelli E, Tonelli C. 2009. A new role for plant R2R3-MYB transcription factors in cell cycle regulation.

Cell Res 19:1231-1232. DOI: 10.1038/cr.2009.123

Devaiah B N, Madhuvanthi R, Karthikeyan A S, Raghothama K G. 2009. Phosphate starvation responses and gibberellic acid biosynthesis are regulated by the MYB62 transcription factor in Arabidopsis. Molecular Plant 2(1), 43-58. DOI: 10.1093/mp/ssn081

Ding Z, Li S, An X, Liu X, Qin H, Wang D. 2008. Transgenic expression of MYB15 confers enhanced sensitivity to abscisic acid and improved drought tolerance in Arabidopsis thaliana. Cell Res 18:1047-1060. DOI: $10.1016 / \mathrm{S} 1673-8527(09) 60003-5$

Du H, Feng B R, Yang SS, Huang YB, Tang YX. 2012a. The R2R3-MYB transcription factor gene family in maize. Plos One 7(6):e37463. DOI: 10.1371/journal.pone.0037463

Du H, Yang SS, Liang Z, Feng BR, Liu L, Huang YB, Tang YX. 2012b. Genome-wide analysis of the MYB transcription factor superfamily in soybean. Bmc Plant Biology 12(1):106. DOI: 10.1186/1471-2229$12-106$

Dubos C, Stracke R, Grotewold E, Weisshaar B, Martin C, Lepiniec L. 2010. MYB transcription factors in Arabidopsis thaliana. Trends in Plant Science 15:573-581. DOI: 10.1016/j.tplants.2010.06.005

Fang Q, Wang Q, Mao H, Xu J, Wang Y, Hu H, He S, Tu JC, Cheng C, Tian GZ, Wang XQ, Liu XP, Zhang C, Luo KM. 2018. AtDIV2, an R-R-type MYB transcription factor of Arabidopsis, negatively regulates salt stress by modulating ABA signaling. Plant Cell Reports 1-13. DOI: 10.1007/s00299-018-2321-6 Finn RD, Coggill P, Eberhardt RY, Eddy SR, Mistry J, Mitchell AL, Potter SC, Punta M, Qureshi M, Sangrador-Vegas A, Salazar GA, Tate J, Batem an A. 2016. The Pfam protein families database: towards a more sustainable future. Nucleic Acids Research 44:279-285. DOI: 10.1093/nar/gkv1344

Gigolashvili T, Yatusevich R, Berger B, Muller C, Flugge U. 2007. The R2R3-MYB transcription factor HAG1/MYB28 is a regulator of methionine-derived glucosinolate biosynthesis in Arabidopsis thaliana. Plant Journal 51(2), 247-261. DOI: 10.1111/j.1365-313X.2007.03133.x 
665 Hou XJ, Li SB, Liu SR, Hu CG, Zhang JZ. 2014. Genome-wide classification and evolutionary and 666 expression analyses of Citrus MYB transcription factor families in sweet orange. PLos One 9(11): e112375. 667 DOI: 10.1371/journal.pone.0112375

668 Hu B, Jin J, Guo AY, He Z, Luo J, Gao G. 2015. GSDS 2.0: an upgraded gene feature visualization server. 669 Bioinformatics 31(8):1296-7. DOI: 10.1093/bioinformatics/btu817

670 Jia L, Clegg MT, Jiang T. 2004. Evolutionary dynamics of the DNA-binding domains in putative R2R3671 MYB genes identified from rice subspecies indica and japonica genomes. Plant Physiol 134: 575-585. DOI:

672

673

674

675

676

677

678

679

680

681

682

683

684

685

686

687 10.1104/pp.103.027201

Jung C, Seo J S, Han SW, Koo Y J, Kim CH, Song S I, Nahm B H, Choi Y D, Cheong J J. 2008 Overexpression of AtMYB44 enhances stomatal closure to confer abiotic stress tolerance in transgenic Arabidopsis. Plant Physiol 146, 623-635. DOI: 10.1104/pp.107.110981

Katiyar A, Smita S, Lenka SK, Rajwanshi R, Chinnusamy V, Bansal KC. 2012. Genome-wide classification and expression analysis of MYB transcription factor families in rice and Arabidopsis. BMC Genomics 13:544. DOI: 10.1186/1471-2164-13-544

Klempnauer KH, Gonda TJ, Bishop JM. 1982. Nucleotide sequence of the retroviral leukemia gene v-myb and its cellular progenitor c-MYB: the architecture of a transduced oncogene. Cell 1982 31:453-463. DOI: 10.1016/0092-8674(82)90138-6

Kou X, Chen Q, Li X, Li M, Kan C, Chen B, Zhang Y, Xue Z. 2015. Quantitative assessment of bioactive compounds and the antioxidant activity of 15 jujube cultivars. Food Chemistry 173:1037-1044. DOI: 10.1016/j.foodchem.2014.10.110

Kranz HD, Denekamp M, Greco R, Jin H, Leyva A, Meissner RC, Petroni K, Urzainqui A, Bevan M, Martin C, Smeekens S, Tonelli C, Paz-Ares J, Weisshaar B. 1998. Towards functional characterisation of the members of the R2R3-MYB gene family from Arabidopsis thaliana. Plant $J$ 16: 263-276. DOI: 10.1046/j.1365-313x.1998.00278.x

Krzywinski M, Schein J, Birol I, Connors J, Gascoyne R, Horsman D, Jones S, Marra M. 2009. Circos: an information aesthetic for comparative genomics. Genome Research 19:1639-1645. DOI: $10.1101 /$ gr.092759.109 
692 Lalitha, S. 2000. Primer premier 5. Biotechnol. Softw. Internet Rep 1, 270-272.DOI:

693

694

695

696

697

698

699

700

701

702

703

704

705

706

707

708

709

710

711

712

713

714

715

716

717

718

\section{$10.1089 / 152791600459894$}

Lam CTW, Gong AGW, Lam KYC, Zhang LM, Chen JP, Dong TTX, Lin HQ, Tsim KWK. 2016. Jujubecontaining herbal decoctions induce neuronal differentiation and the expression of anti-oxidant enzymes in cultured PC12 cells. Journal of Ethnopharmacology 188:275-283. DOI: 10.1016/j.jep.2016.05.015

Li J, Yang X, Wang Y, Li X, Gao Z, Pei M, Chen Z, Qu LJ, Gu H. 2006. Two groups of MYB transcription factors share a motif which enhances trans-activation activity. Biochemical \& Biophysical Research Communications 341(4):1155-1163. DOI: 10.1016/j.bbrc.2006.01.077

Li JW, Fan LP, Ding SD, Ding XL. 2007. Nutritional composition of five cultivars of Chinese jujube. Food Chemistry 103(2):454-460. DOI: 10.1016/j.foodchem.2006.08.016

Li KT, Zhang J, Kang YH, Chen MC, Song TT, Geng H, Tian J, Yao YC. 2018. McMYB10 Modulates the Expression of a Ubiquitin Ligase, McCOP1 During Leaf Coloration in Crabapple. Front Plant Sci 9:704. DOI: $10.3389 /$ fpls.2018.00704

Li X, Xue C, Li J, Qiao X, Li L, Yu L, Huang Y, Wu J. 2016. Genome-wide identification, evolution and functional divergence of MYB transcription factors in Chinese white pear (Pyrus bretschneideri). Plant and Cell Physiology 57:824-847. DOI: 10.1093/pcp/pcw029

Li ZJ, Peng RH, Tian YS, Han HJ, Xu J, Yao QH. 2016. Genome-wide identification and analysis of the MYB transcription factor superfamily in Solanum lycopersicum. Plant and Cell Physiology 57(8):16571677. DOI: 10.1093/pcp/pcw091

Lipsick JS. 1996. One billion years of Myb. Oncogene 13:223-235. PMID:8710361

Liu C, Xie T, Chen C, Luan A, Long J, Li C, Ding Y, He Y. 2017. Genome-wide organization and expression profiling of the R2R3-MYB transcription factor family in pineapple (Ananas comosus). BMC Genomics 18(1):503. DOI: 10.1186/s12864-017-3896-y

Liu LS, White MJ, MacRae TH. 1999. Transcription factors and their genes in higher plants: functional domains, evolution and regulation. European Journal of Biochemistry 262:247-257. PMID:10336605

Liu MJ, Zhao J, Cai QL, Liu GC, Wang JR, Zhao ZH, Liu P, Dai L, Yan GJ, Wang WJ, Li XS, Chen Y, Sun YD, Liu ZG, Lin MJ, Xiao J, Chen YY, Li XF, Wu Bin, Ma Y, Jian JB, Yang W, Yuan Z, Sun XC, 
Wei YL, Yu LL, Zhang C, Liao SG, He RJ, Guang XM, Wang Z, Zhang YY, Luo LH. 2014. The complex jujube genome provides insights into fruit tree biology. Nat Commun 5:5315. DOI: 10.1038/ncomms6315 Liu Y, Shi Z, Maximova SN, Payne MJ, Guiltinan MJ. 2015. Tc-MYBPA an Arabidopsis TT2-like transcription factor and functions in the regulation of proanthocyanidin synthesis in Theobroma cacao. BMC Plant Biology 15(1):160. DOI: 10.1186/s12870-015-0529-y

Liu Z, Zhang L, Xue C, Fang H, Zhao J, Liu MJ. 2017. Genome-wide identification and analysis of MAPK and MAPKK gene family in Chinese jujube (Ziziphus jujuba Mill.). Bmc Genomics 18(1):855. DOI: $10.1186 / \mathrm{s} 12864-017-4259-4$

Livak KJ, Schmittgen TD. 2001. Analysis of relative gene expression data using real-time quantitative PCR and the 2- $\Delta \Delta \mathrm{CT}$ method. Methods 25: 402-408. DOI: 10.1006/meth.2001.1262

Mmadi MA, Dossa K, Wang LH, Zhou R, Wang YY, Cisse N, Sy MO, Zhang XR. 2017. Functional characterization of the versatile MYB gene family uncovered their important roles in plant development and responses to drought and waterlogging in sesame. Genes 8:362-380. DOI: 10.3390/genes8120362 Ogata K, Morikawa S, Nakamura H, Sekikawa A, Inoue T, Kanai H, Sarai A, Ishii S, Nishimura Y. 1994. Solution structure of a specific DNA complex of the Myb DNA-binding domain with cooperative recognition helices. Cell 79(4):639-48. DOI: 10.1016/0092-8674(94)90549-5

Ogata K, Kaneiishii C, Sasaki M, Hatanaka H, Nagadoi A, Enari M, Nakamura H, Nishimura Y, Ishii S, Sarai A. 1996. The cavity in the hydrophobic core of Myb DNA-binding domain is reserved for DNA recognition and trans-activation. Nature Structural Biology 3:178-187. DOI: 10.1038/nsb0296-178

Paz-Ares J, Ghosal D, Wienand U, Peterson P, Saedler H. 1987. The regulatory c1 locus of Zea mays encodes a protein with homology to MYB oncogene products and with structural similarities to transcriptional activators. EMBO Journal 6:3553-3558. DOI: 10.1002/j.1460-2075.1987.tb02684.x Puche LM, Cumplido-Laso G, Amil-Ruiz F, Hoffmann T, Ring L, Rodríguez-Franco A, Caballero JL, Schwab W, Muñoz-Blanco J, Blanco-Portales R. 2014. MYB10 plays a major role in the regulation of flavonoid/phenylpropanoid metabolismduring ripening of Fragaria $\times$ ananassa fruits. Journal of Experimental Botany 65(2):401-417. DOI: 10.1093/jxb/ert377

Raffaele S, Vailleau F, Leger A, Joubes J, Miersch O, Huard C, Blee E, Mongrand S, Domergue F, Roby 
746

747

748

749

750

751

752

753

754

755

756

757

758

759

760

761

762

763

764

765

766

767

768

769

770

771

772

D. 2008. A MYB transcription factor regulates very-long-chain fatty acid biosynthesis for activation of the hypersensitive cell death response in Arabidopsis. The Plant Cell 20(3), 752-767. DOI: 10.1105/tpc.107.054858

Riechmann JL, Heard J, Martin G, Reuber L, Jiang CZ, Keddie J, Adam L, Pineda O, Ratcliffe OJ, Samaha RR, Creelman R, Pilgrim M, Broun P, Zhang JZ, Ghandehari D, Sherman BK, Yu G. 2000. Arabidopsis transcription factors: genome-wide comparative analysis among eukaryotes. Science 290:2105-2110. PMID: 11118137

Saha G, Park JI, Ahmed NU, Kayum MA, Kang KK, Nou IS. 2016. Characterization and expression profiling of MYB transcription factors against stresses and during male organ development in Chinese cabbage (Brassica rapa ssp. pekinensis). Plant Physiology and Biochemistry 104:200-215. DOI: 10.1016/j.plaphy.2016.03.021

Salih H, Gong W, He S, Sun G, Sun J, Du X. 2016. Genome-wide characterization and expression analysis of MYB transcription factors in Gossypium hirsutum. BMC Genet 17:129. DOI: 10.1186/s12863-016-04368

Shao F, Qiang L, Wilson I W, Qiu D. 2017. Genome-wide identification and characterization of the SPL, gene family in Ziziphus jujuba. Gene 627:315-321. DOI: 10.1016/j.gene.2017.06.044

Shin B, Choi G, Yi H, Yang S, Cho I, Kim J, Lee S, Paek NC, Kim JH, Song PS, Choi G. 2002. AtMYB21, a gene encoding a flower-specific transcription factor, is regulated by COP1. Plant Journal 30(1):23-32. DOI: 10.1046/j.1365-313X.2002.01264.X

Shin R, Burch A Y, Huppert K, Tiwari, S B, Murphy, A S, Guilfoyle T J, Schachtman D P. 2007. The Arabidopsis Transcription Factor MYB77 Modulates Auxin Signal Transduction. The Plant Cell 19(8), 2440-2453. DOI: $10.1105 /$ tpc. 107.050963

Singh K, Foley RC, Onate-Sanchez L. 2002. Transcription factors in plant defense and stress response. Current Opinion in Plant Biology 5(5):430-436. DOI: 10.1016/S1369-5266(02)00289-3

Song S, Zhou H, Sheng S, Cao M, Li Y, Pang X. 2017. Genome-Wide Organization and Expression Profiling of the SBP-Box Gene Family in Chinese Jujube (Ziziphus jujuba Mill.). International Journal of Molecular Sciences 18(8):1734. DOI: 10.3390/ijms 18081734 
773 Stracke R, Ishihara H, Huep G, Barsch A, Mehrtens F, Niehaus K, Weisshaar B. 2010. Differential

774 regulation of closely related R2R3-MYB transcription factors controls flavonol accumulation in different

775 parts of the Arabidopsis thaliana seedling. Plant Journal 50(4):660-677. DOI: 10.1111/j.1365-

$776 \quad 313 X .2007 .03078 . x$

777 Stracke R, Werber M, Weisshaar B. 2001. The R2R3-MYB gene family in Arabidopsis thaliana. Current 778 Opinion in Plant Biology 4:447-456. DOI: 10.1016/S1369-5266(00)00199-0

779 Swarbreck D, Wilks C, Lamesch P, Berardini TZ, Garcia-Hernandez M, Foerster H, Li DH, Meyer T, 780 Muller R, Ploetz L, Radenbaugh A, Singh S, Swing V, Tissier C, Zhang P and Huala E. 2008. The Arabidopsis Information Resource (TAIR): gene structure and function annotation. Nucleic Acids Res 36:D1009-D1014. DOI: 10.1093/nar/gkm965

Tamura K, Stecher G, Peterson D, Filipski A, Kumar S. 2013. MEGA6: molecular evolutionary genetics analysis version 6.0. Molecular Biology \& Evolution 30, 2725e2729. DOI: 10.1093/molbev/mst197 Wang N, Qu C Jiang S, Chen Z, Xu H, Fang H, Su M, Zhang J, Wang Y, Liu W, Zhang Z, Lu N, Chen X. 2018. The proanthocyanidin-specific transcription factor MdMYBPA1 initiates anthocyanin synthesis under low temperature conditions in red-fleshed apple. Plant journal. DOI: 10.1111/tpj.14013

Wang Y, Tang H, DeBarry JD, Tan X, Li J, Wang X, Lee T-h, Jin H, Marler B, Guo H. 2012. MCScanX: a toolkit for detection and evolutionary analysis of gene synteny and collinearity. Nucleic Acids Res 40(7):e49. DOI: 10.1093/nar/gkr1293

Wang YG, Xu Y, Ma XP, Liu XF, Yang MJ, Fan WG, Ren HW, Efehi N, Wang XL, Zhu XQ. 2018.

Extraction, purification, characterization and antioxidant activities of polysaccharides from Zizyphus jujuba cv. Linzexiaozao. International Journal of Biological Macromolecules 118(Pt B):2138-2148. DOI: 10.1016/j.ijbiomac.2018.07.059

Wei X, Liu K, Zhang Y, Feng Q, Wang L, Zhao Y, Li D, Zhao Q, Zhu X, Zhu X, Li W, Fan D, Gao Y, Lu Y, Zhang X, Tang X, Zhou C, Zhu C, Liu L, Zhong R, Tian Q, Wen Z, Weng Q, Han B, Huang X, Zhang X. 2015. Genetic discovery for oil production and quality in sesame. Nature Communication 6:8609-8622. DOI: $10.1038 /$ ncomms 9609

Weston K. 1998. Myb proteins in life, death and differentiation. Curr Opin Genet Dev 8: 76-81. DOI: 
800

801

802

803

804

805

806

807

808

809

810

811

812

813

814

815

816

817

818

819

820

821

822

823

824

825

\subsection{6/S0959-437X(98)80065-8}

Wu CS, Gao QH, Guo XD, Yu JG, Wang M. 2012. Effect of ripening stage on physicochemical properties and antioxidant profiles of a promising table fruit 'pear-jujube' (Zizyphus jujuba Mill.). Scientia Horticulturae 148(148):177-184. DOI: 10.1016/j.scienta.2012.09.026

Xing C, Liu Y, Zhao L, Zhang S, Huang X. 2018. A novel MYB Transcription Factor regulates AsA synthesis and effects cold tolerance. Plant Cell \& Environmen. DOI: 10.1111/pce.13387

Yu Y, Zhang S, Ren Y, Li H, Zhang X, Di J. 2012. Jujube preservation using chitosan film with nanosilicon dioxide. Journal of Food Engineering 113(3):408-414. DOI: 10.1016/j.jfoodeng.2012.06.021

Yuan CG, Huo C, Gui B, Liu JF, Chen YS. 2017. Facile phyto-mediated synthesis of silver nanoparticles using chinese winter jujube (Ziziphus jujuba Mill. cv. Dongzao) extract and their antibacterial/catalytic properties. Iet Nanobiotechnology 11(8):973-980. DOI: 10.1049/iet-nbt.2016.0242

Zhang C, Bian Y, Hou S, Li X. 2018. Sugar transport played a more important role than sugar

biosynthesis in fruit sugar accumulation during Chinese jujube domestication. Planta. DOI:

$10.1007 / \mathrm{s} 00425-018-2971-1$

Zhang C, Ma R, Xu J, Yan J, Guo L, Song J, Feng R, Yu M. 2018. Genome-wide identification and classification of MYB superfamily genes in peach. Plos One 13(6):e0199192. DOI: 10.1371/journal.pone.0199192

Zhang L, Zhao J, Feng C, Liu M, Wang J, Hu Y. 2017. Genome-wide identification, characterization of the MADS-box gene family in Chinese jujube and their involvement in flower development. Scientific Reports 7(1):1025. DOI: $10.1038 / \mathrm{s} 41598-017-01159-8$

Zhao ZH, Liu MJ, Tu PF. 2008 Characterization of water soluble polysaccharides from organs of Chinese Jujube (Ziziphus jujuba Mill. cv. Dongzao). European Food Research \& Technology 226(5):985-989.

DOI: $10.1007 / \mathrm{s} 00217-007-0620-1$

Zhou C, Chen Y, Wu Z, Lu W, Han J, Wu P, Chen Y, Li M, Jiang H, Wu G. 2015. Genome-wide analysis of the MYB gene family in physic nut (Jatropha curcas L.). Gene 572(1):63-71. DOI: 10.1016/j.gene.2015.06.072. 


\section{Figure 1}

Phylogenetic tree of the R2R3-MYB, R1R2R3-MYB and 4R-MYB subfamilies of jujube and Arabidopsis and conserved motif and gene structure analysis of the R2R3-MYB and R1R2R3-MYB subfamily proteins of jujube.

(A) Phylogenetic tree of the MYB proteins of jujube and Arabidopsis. The sequences of the 171 MYB superfamily proteins of jujube and 132 Arabidopsis R2R3-MYB, R1R2R3-MYB and 4RMYB proteins were aligned by ClustalW, and the phylogenetic tree was constructed using MEGA 6. The small white triangles represent the 171 jujube MYB proteins, and the small black triangles represent the 132 Arabidopsis MYB proteins. The names of each group are marked by English letters with Arabic numbers. (B) Distributions of conserved motifs in ZjMYB genes. The motifs of numbers 1-15 are indicated in different colored boxes. The sequence information of the motifs is provided in Table S2. (C) The exon-intron structure of jujube MYB genes. The yellow boxes and black lines indicate exons and introns, respectively. The green boxes at the two ends of the sequences indicate upstream and downstream regions, respectively. The MYB domains are highlighted by red boxes. 


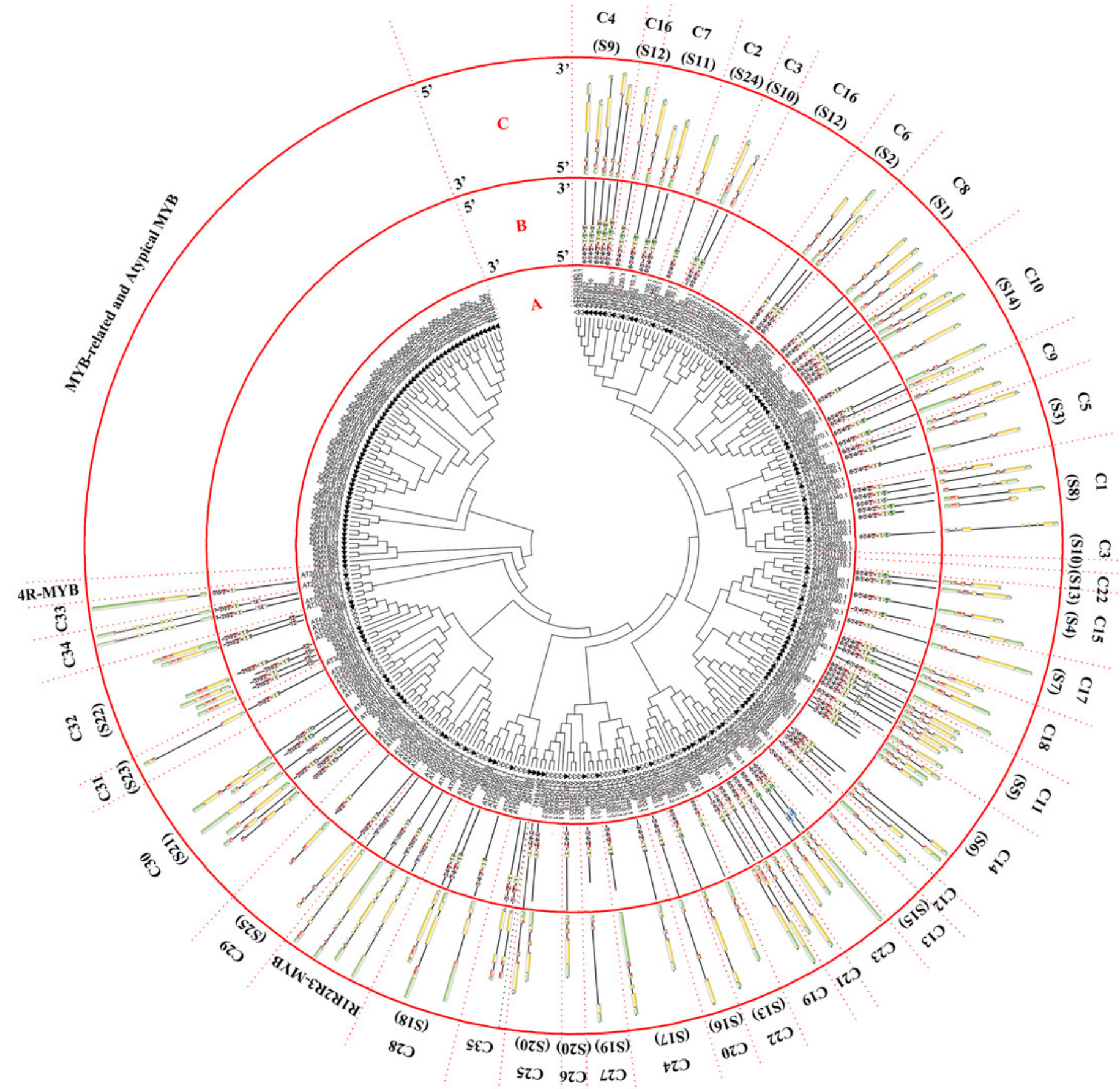




\section{Figure 2}

Phylogenetic tree of the MYB-related (1R-MYB) and atypical MYB subfamilies of jujube and Arabidopsis, and conserved motif and gene structure analysis of MYB-related and atypical MYB subfamily proteins of jujube.

(A) Phylogenetic tree of the MYB proteins of jujube and Arabidopsis. The sequences of the 58 MYB-related and 9 atypical MYB proteins of jujube with the 60 MYB-related and 6 atypical MYB proteins of Arabidopsis were aligned by ClustalW, and the phylogenetic tree was constructed using MEGA 4. The small white triangles represent the 67 jujube MYB proteins and the small black triangles represent the 66 Arabidopsis MYB proteins. The names of each group are marked by English letters with Arabic numbers. (B) Distributions of conserved motifs in ZjMYB genes. The motifs of numbers 1-15 are indicated in different colored boxes. The sequence information of the motifs is provided in Table S3. (C) The exon-intron structure of jujube MYB genes. The yellow boxes and black lines indicate exons and introns, respectively. The green boxes at the two ends of the sequences indicate upstream and downstream regions, respectively. The MYB domains are highlighted by red boxes. 


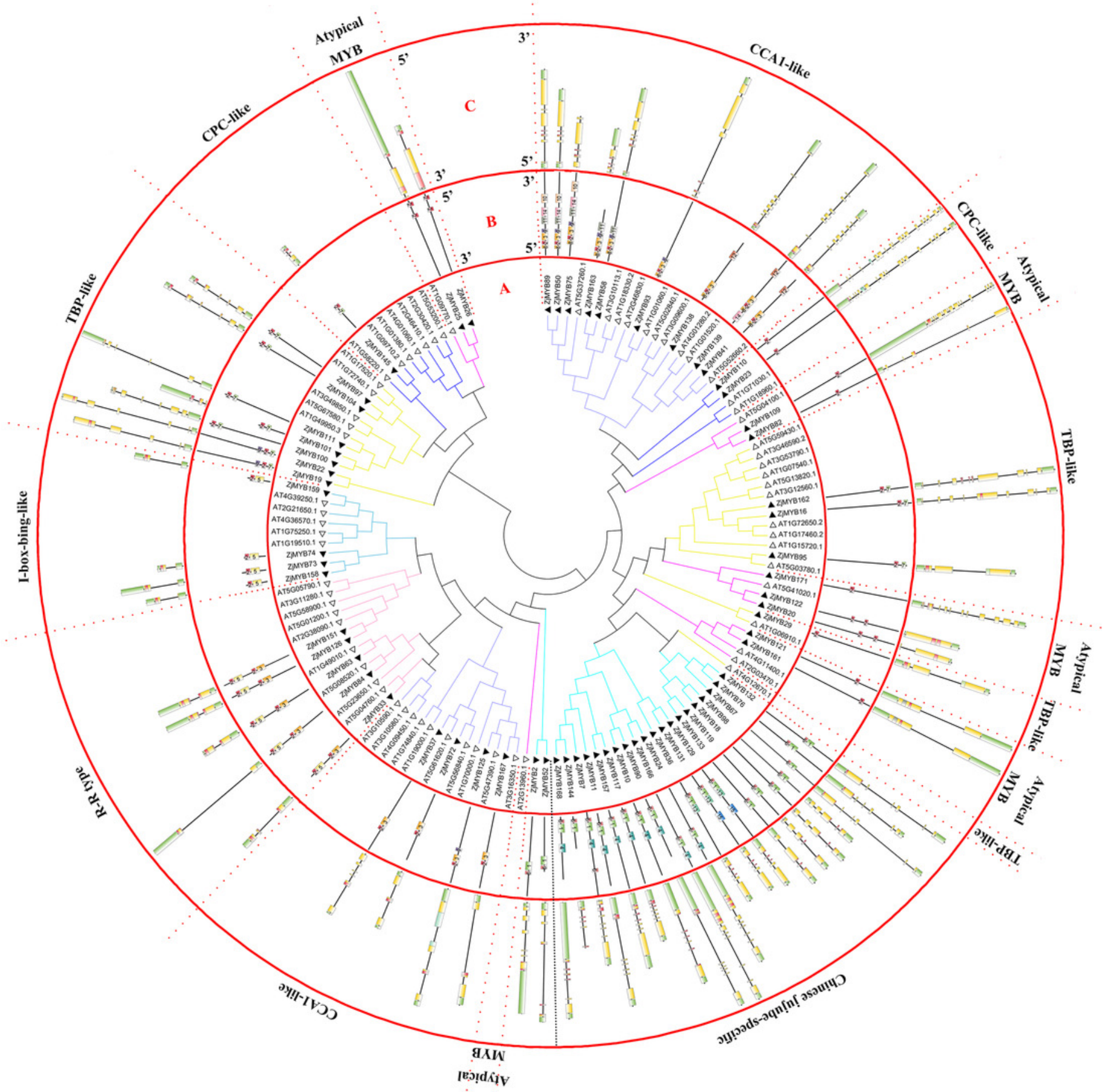


Figure 3

R2 and R3 MYB repeats of the proteins of R2R3-MYB subfamily in jujube.

The logo sequences of R2 and R3 repeats were composed of motifs 3, 4, 2 and 1 in jujube. The overall height of each stack showed the conservation of the MYB protein sequence at that position. English letters indicate the different type of amino acid residue.

$\mathrm{R} 2$ repeat

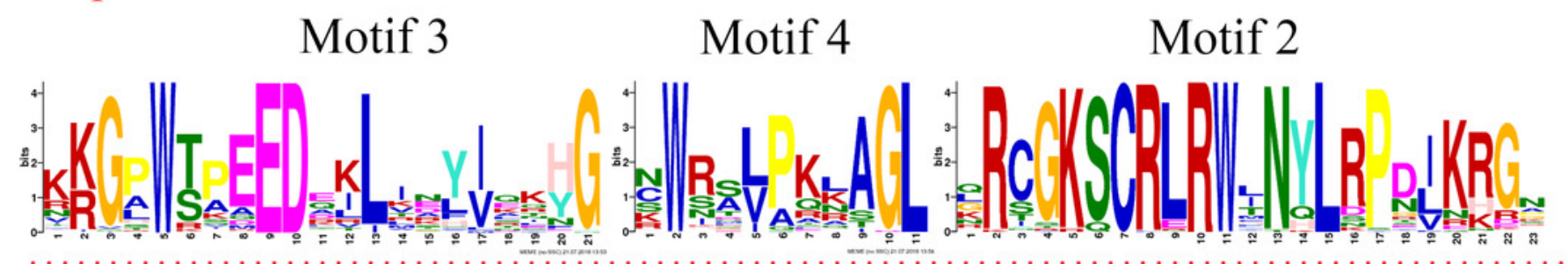

$\mathrm{R} 3$ repeat

Motif 2 (continued) Motif 1

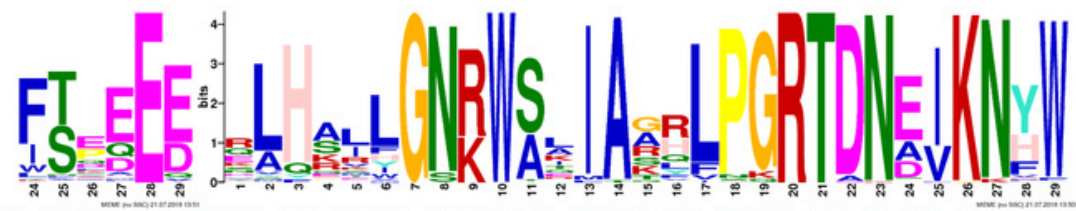




\section{Figure 4}

R1, R2 and R3 MYB repeats of the proteins of R1R2R3-MYB subfamily in jujube.

The logo sequences of R1, R2 and R3 repeats were composed of motifs $9,3,10,2,1$ and 7 in jujube. The overall height of each stack showed the conservation of the MYB protein sequence at that position. English letters indicate the different type of amino acid residue.

$\mathrm{R} 1$ repeat

Motif 9

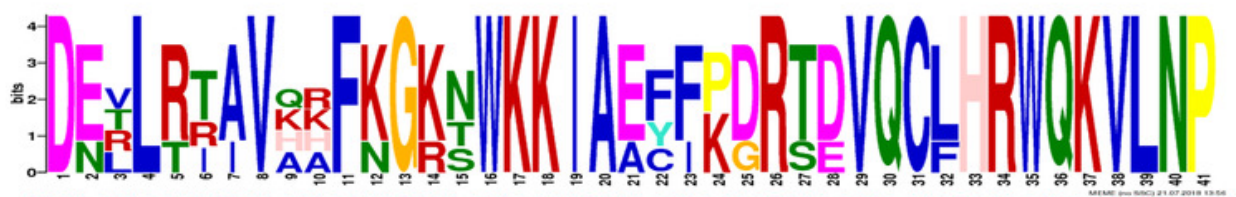

R2 repeat

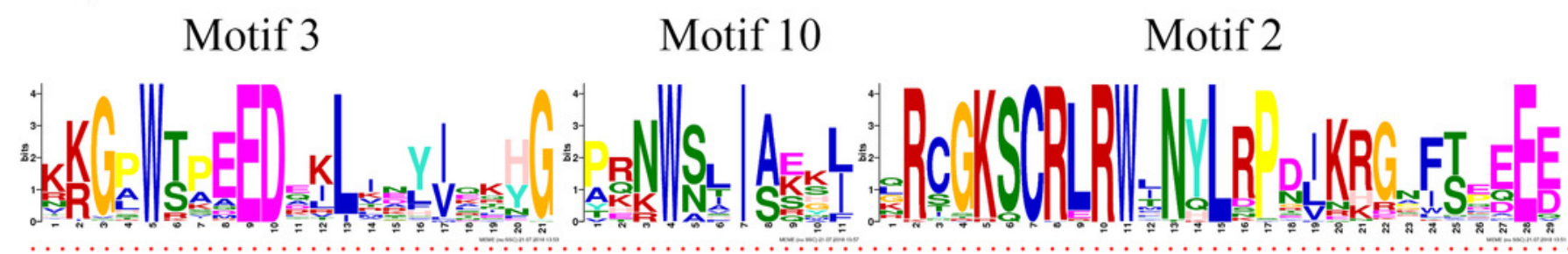

$\mathrm{R} 3$ repeat

Motif 1

Motif 7

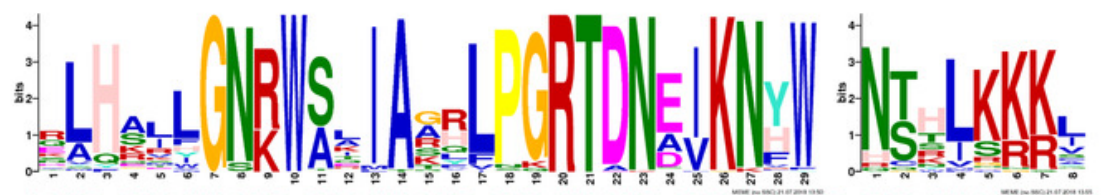


Figure 5

This logo indicates the sequence similarities of the ZjMYB repeats of the R-R-type, CCA 1-like and TBP-like MYB-related proteins.

R-R (A) was the first repeat

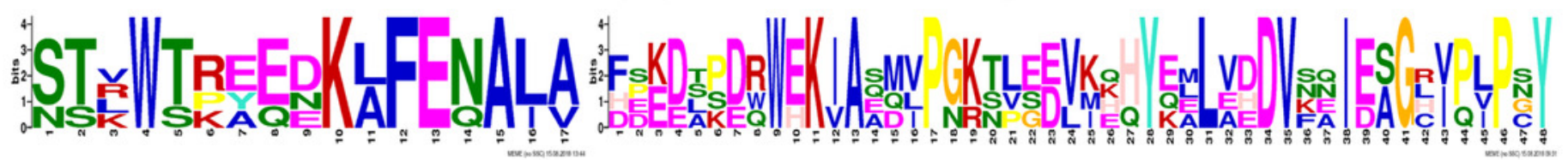

$\mathrm{R}-\mathrm{R}$ (B) was the second repeat

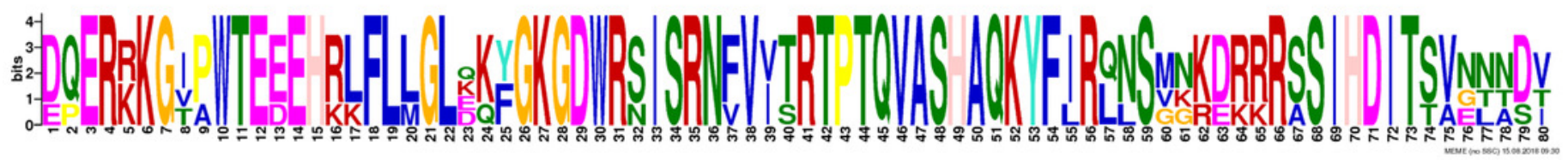

CCA1-like (clade II)

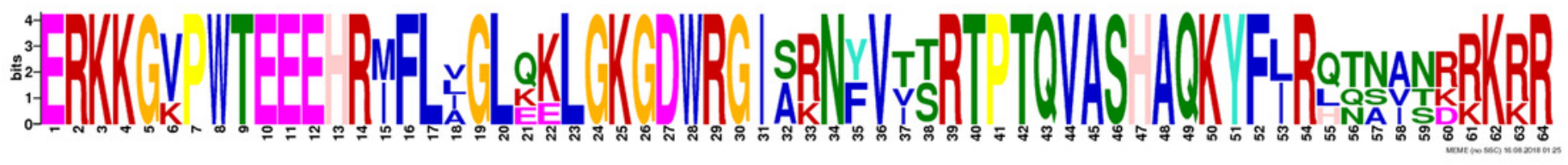

CCA1-like (clade I)

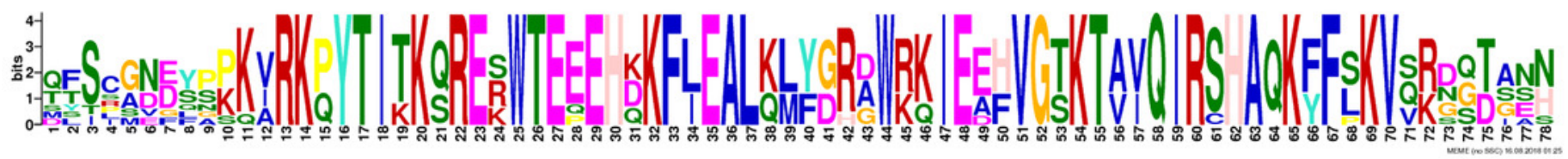

TBP-like

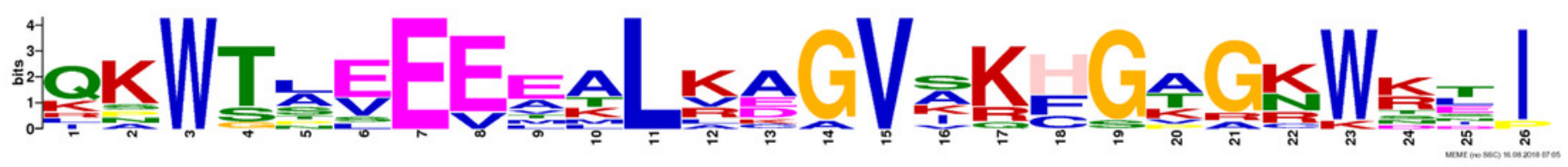




\section{Figure 6}

Motif and gene structure analysis of the 4R-MYB protein.

(A) Distributions of conserved motifs in 4R-MYB genes of Arabidopsis and jujube. The motifs of numbers 1-15, are indicated in different colored boxes. The sequence information of motifs had provided in Table S4. (B) Exon-intron structure of 4R-MYB in jujube. The yellow boxes and black lines indicate exons and introns, respectively. Green boxes at the two ends of sequences indicate upstream and downstream regions. The MYB domains are highlighted by red boxes.

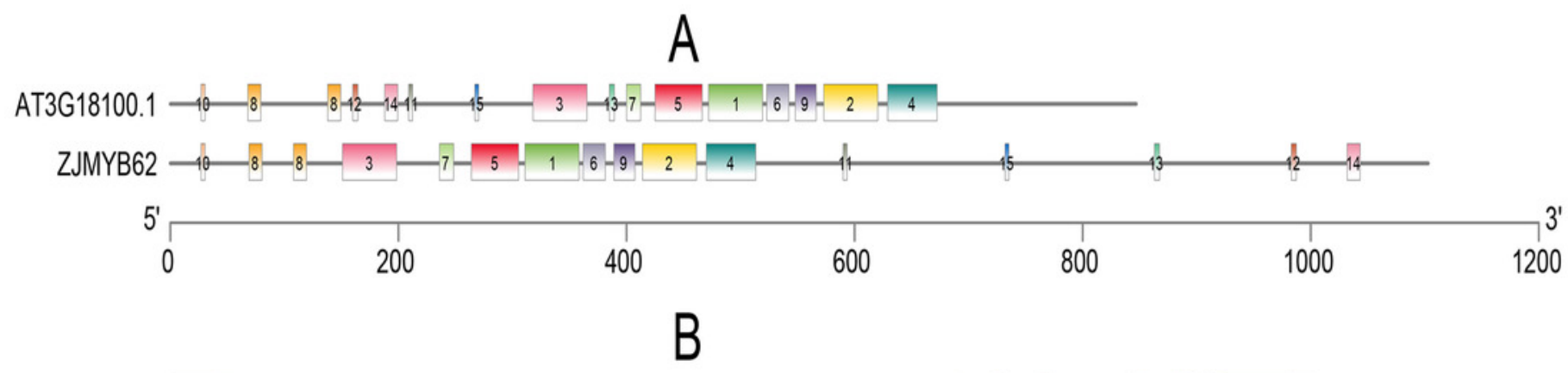

ZJMYB62

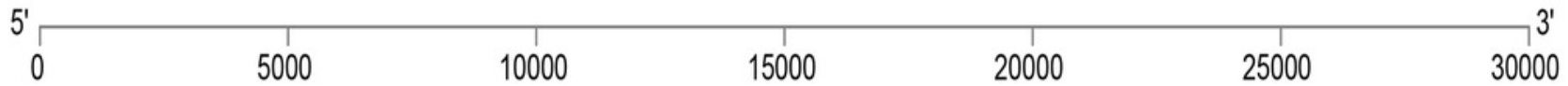




\section{Figure 7}

Chromosomal distribution and gene duplication of jujube MYB superfamily genes.

The inner ring denotes the 12 pseudochromosomes of jujube. The positions of the labels in the optical map are shown by the red bar (a higher bar corresponds to greater density). The outer ring shows the chromosomal distribution of jujube MYB superfamily genes. The scale is $5 \mathrm{Mb}$. The three yellow lines inside the circle are the tandem duplicated MYB gene pairs. 


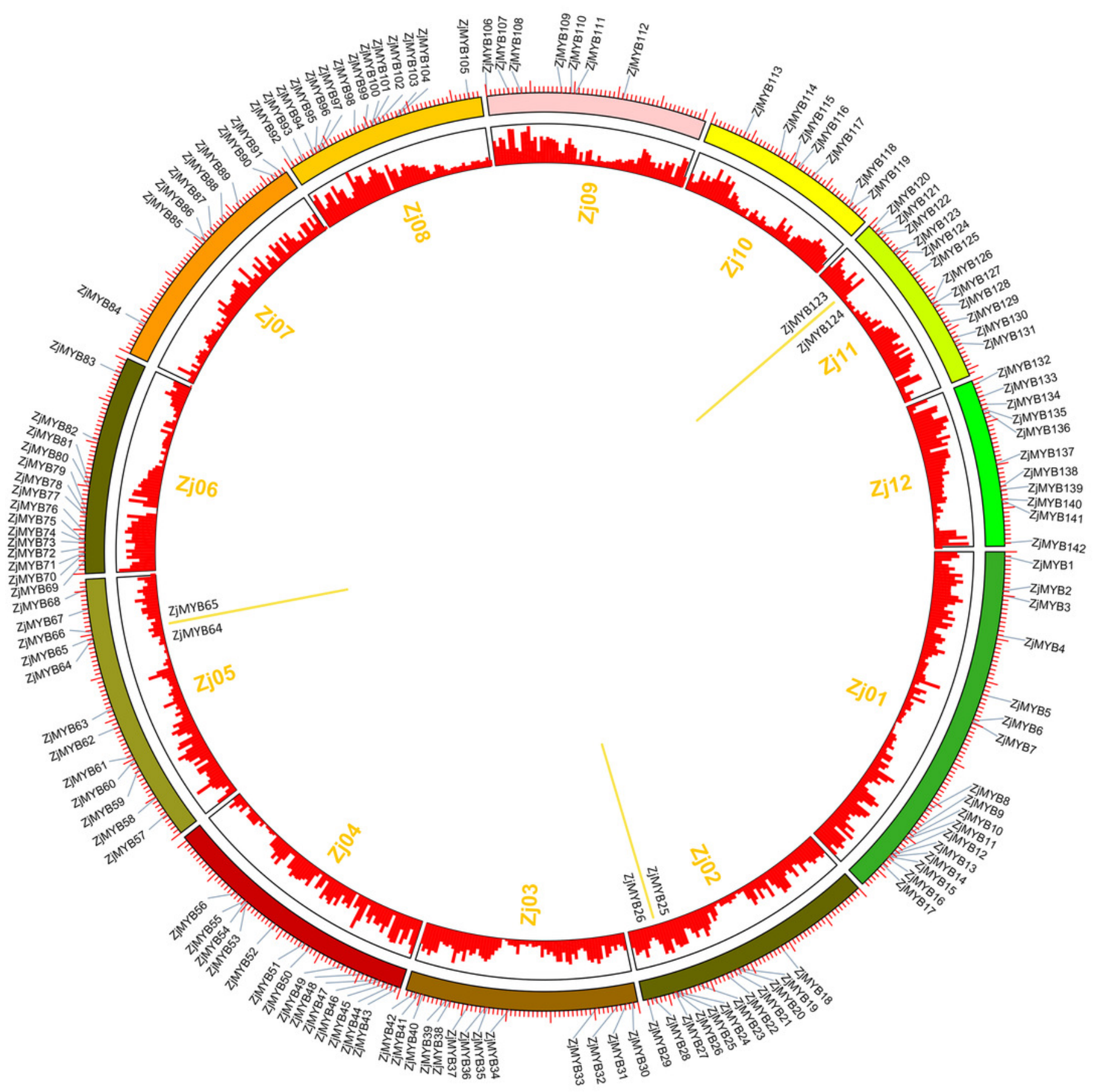




\section{Figure 8}

Heatmap of the expression levels of MYB genes in different developmental stages in jujube fruit and the expression levels of 10 ZjMYB genes by qRT-PCR.

Y (young stage), EN (enlargement stage), WM (white mature stage), HR (half-red stage) and FR (full-red stage). The color scale shown at the top represents log2-transformed FPKM (fragments per kilobase of exon per million mapped reads) counts. Original FPKM counts are displayed in the corresponding rectangles. Red indicates high expression and green indicates low expression. We eliminated the jujube MYB genes whose expression levels were 0 in all stages. (A) Heatmap of the expression levels of 71 R2R3-MYB, 3 R1R2R3-MYB and 1 4R-MYB genes in different developmental stages in jujube fruit. (B) Heatmap of the expression levels of 44 MYB-related and 7 atypical MYB genes in different developmental stages in jujube fruit. A1-A9 and B1-B8 indicate the different subgroups. (C) Expression analysis of 10 MYB genes in jujube by qRT-PCR. The data were normalized to the UBQ gene, and the vertical bars indicate the standard deviation. 

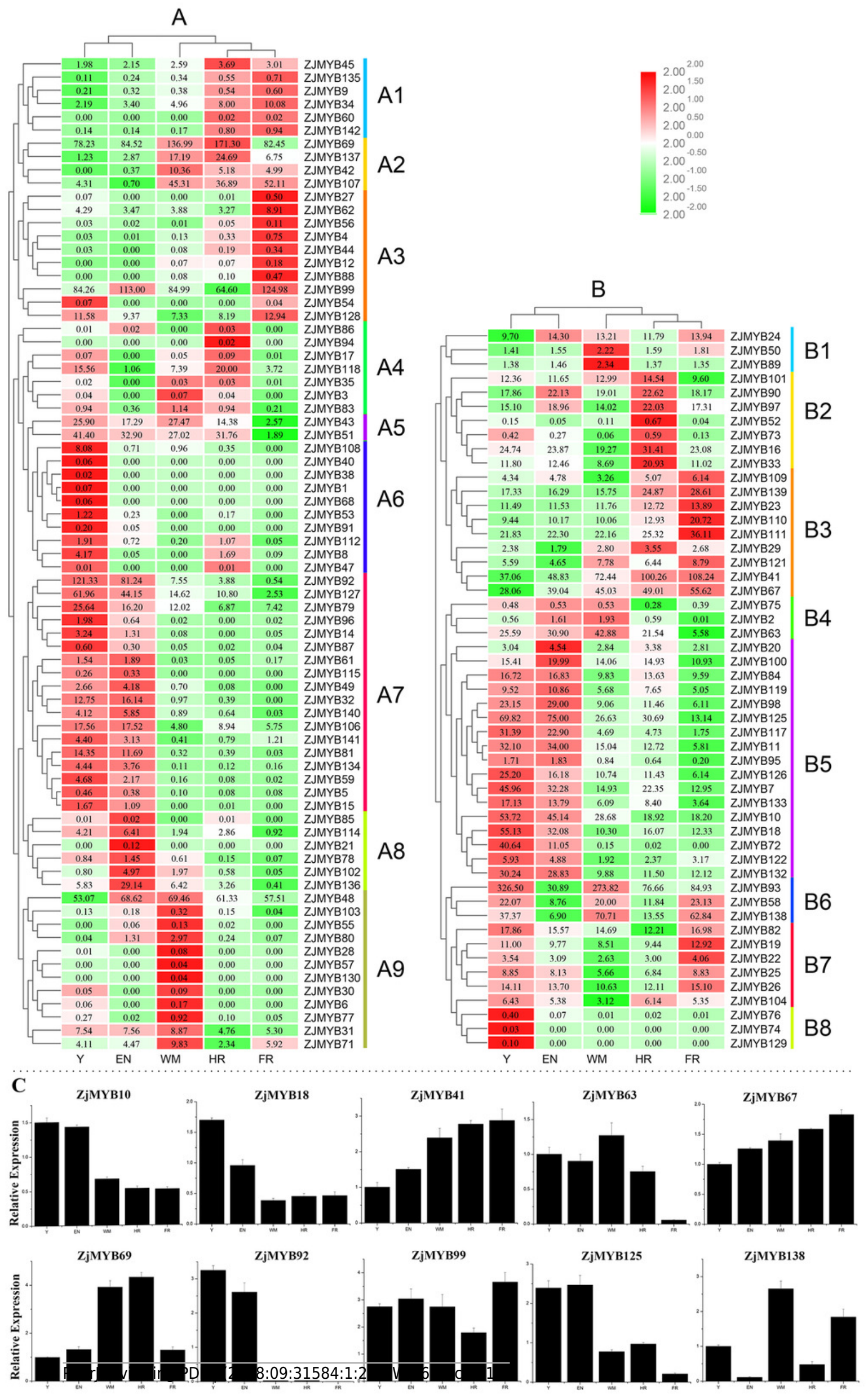
Figure 9

Total flavonoids content of fruit development stages of jujube.

The results were represented as the mean \pm standard deviation. Y (young stage), EN (enlargement stage), WM (white mature stage), HR (half-red stage) and FR (full-red stage).

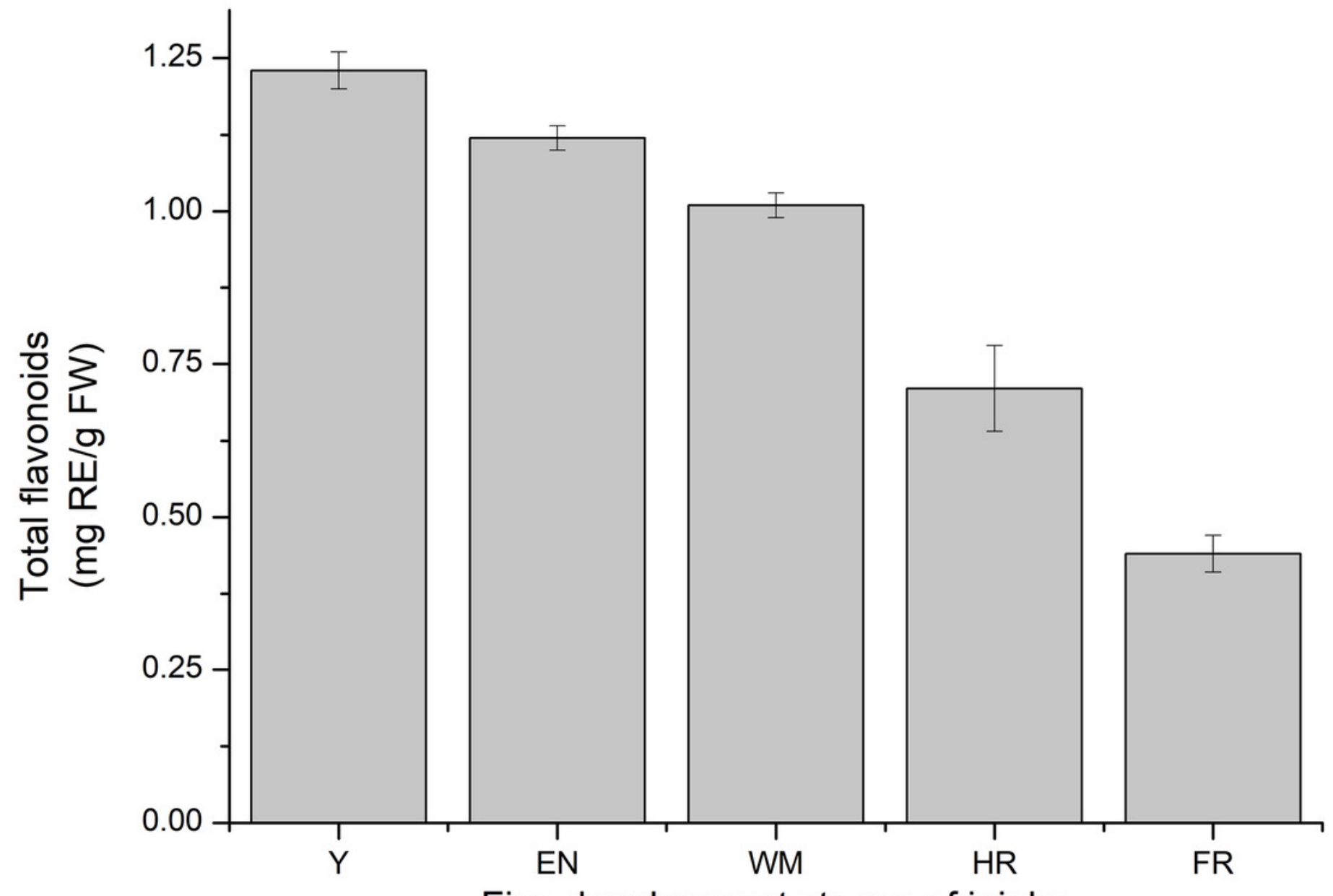

Five development stages of jujube 


\section{Figure 10}

The correlation analysis of total flavonoid content with ZjMYB gene expression in jujube fruit.

The correlation analysis was performed using the original FPKM counts and the expression levels of the 93 MYB genes and the total flavonoid contents during the 5 developmental stages in jujube fruit. The " $x$ " symbol indicates that the correlation is not significant at the 0.05 level. Yellow and red circles indicate that the correlation is significant and positive at the 0.05 level, and the green and blue circles indicate that the correlation is significant and negative at the 0.05 level. The correlations between total flavonoid contents and MYB genes are marked in the 2 red boxes. (A) The correlation analysis of total flavonoid contents with the 42 MYB-related and 7 atypical MYB genes. (B) The correlation analysis of total flavonoid contents with the 40 R2R3-MYB, 3 R1R2R3-MYB and 1 4R-MYB genes.

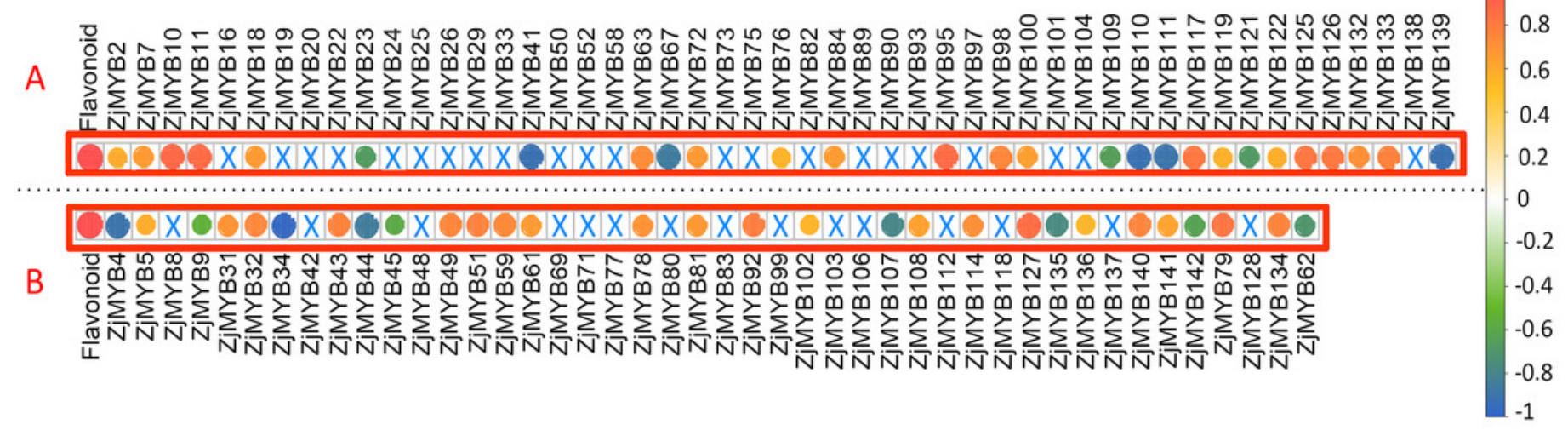




\section{Figure 11}

Synteny analysis of MYB genes between jujube and two other plant species (peach and Arabidopsis).

Twelve chromosomes of jujube (Zj01-Zj12) and five chromosomes of Arabidopsis (A01-A05), as well as eight chromosomes of peach (Pp01-Pp08), were mapped in different colors. The gray lines in the background indicate the collinear blocks within jujube and other plant genomes. The red lines connecting the MYB genes in jujube indicate paralogous MYB gene pairs. The green and blue lines connect the MYB genes in jujube and Arabidopsis and in jujube and peach that were orthologous, respectively. 


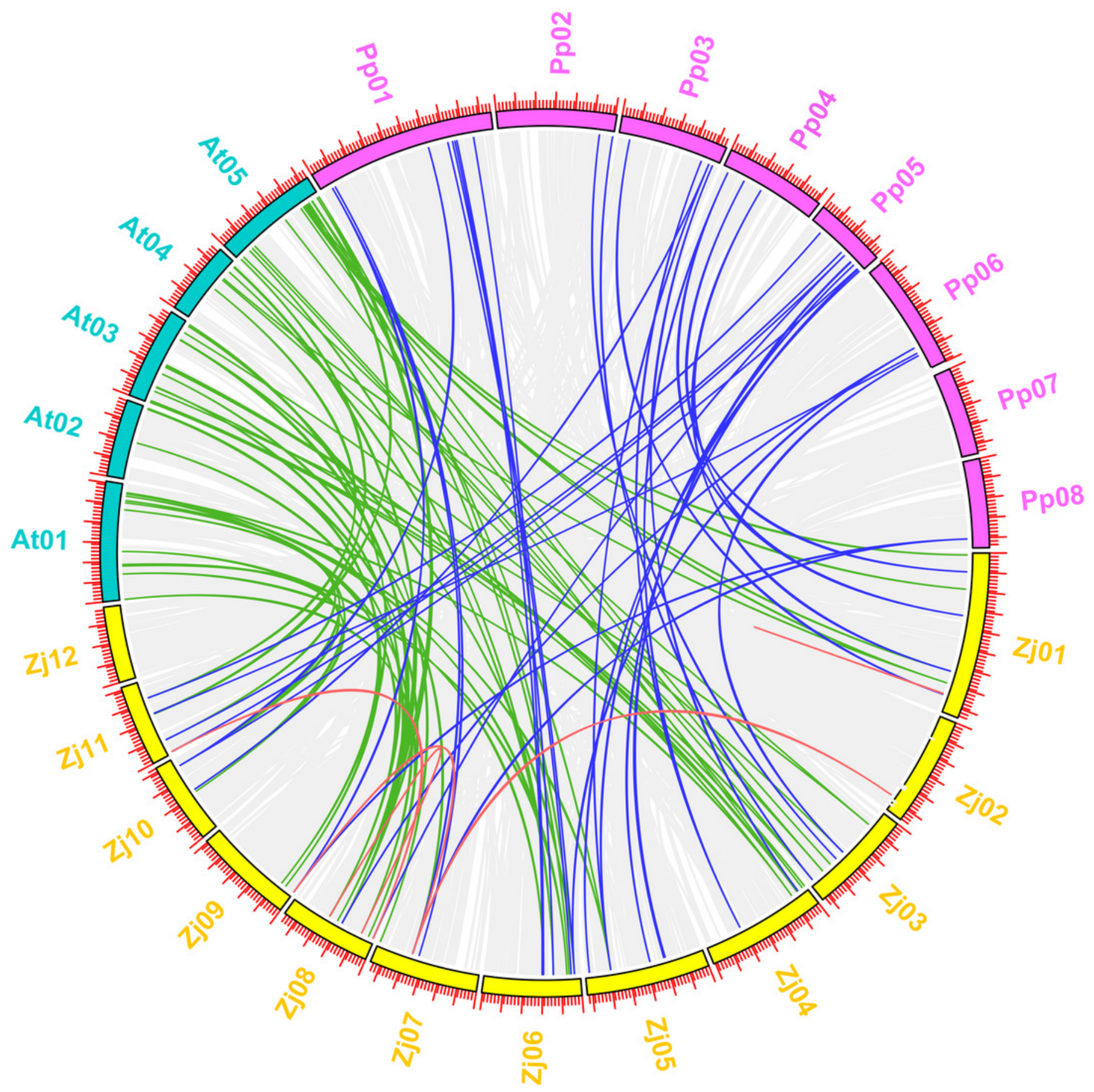

